\title{
Huntington's disease brain-derived small RNAs recapitulate associated neuropathology in mice
}

\author{
Jordi Creus-Muncunill ${ }^{1,2,3,9} \cdot$ Anna Guisado-Corcoll ${ }^{1,2,3} \cdot$ Veronica Venturi $^{4} \cdot$ Lorena Pantano $^{5}$. Georgia Escaramís ${ }^{1,6}$. \\ Marta García de Herreros ${ }^{1,2,3}$ - Maria Solaguren-Beascoa ${ }^{1}$. Ana Gámez-Valero ${ }^{1}$. Cristina Navarrete ${ }^{4}$. \\ Mercè Masana ${ }^{1,2,3}$ • Franc Llorens ${ }^{3,7,8}$ • Daniela Diaz-Lucena ${ }^{3,7} \cdot$ Esther Pérez-Navarro ${ }^{1,2,3}$ • Eulàlia Martí 1,4,6
}

Received: 12 January 2021 / Revised: 19 January 2021 / Accepted: 20 January 2021

(c) The Author(s), under exclusive licence to Springer-Verlag GmbH, DE part of Springer Nature 2021

\begin{abstract}
Progressive motor alterations and selective death of striatal medium spiny neurons (MSNs) are key pathological hallmarks of Huntington's disease (HD), a neurodegenerative condition caused by a CAG trinucleotide repeat expansion in the coding region of the huntingtin (HTT) gene. Most research has focused on the pathogenic effects of the resultant protein product(s); however, growing evidence indicates that expanded CAG repeats within mutant HTT mRNA and derived small CAG repeat RNAs (sCAG) participate in HD pathophysiology. The individual contribution of protein versus RNA toxicity to HD pathophysiology remains largely uncharacterized and the role of other classes of small RNAs (sRNA) that are strongly perturbed in HD is uncertain. Here, we demonstrate that sRNA produced in the putamen of HD patients (HD-sRNA-PT) are sufficient to induce HD pathology in vivo. Mice injected with HD-sRNA-PT show motor abnormalities, decreased levels of striatal HD-related proteins, disruption of the indirect pathway, and strong transcriptional abnormalities, paralleling human HD pathology. Importantly, we show that the specific blockage of sCAG mitigates HD-sRNA-PT neurotoxicity only to a limited extent. This observation prompted us to identify other sRNA species enriched in HD putamen with neurotoxic potential. We detected high levels of tRNA fragments (tRFs) in HD putamen, and we validated the neurotoxic potential of an Alanine derived tRF in vitro. These results highlight that HD-sRNA-PT are neurotoxic, and suggest that multiple sRNA species contribute to striatal dysfunction and general transcriptomic changes, favoring therapeutic strategies based on the blockage of sRNA-mediated toxicity.
\end{abstract}

Keywords RNA toxicity $\cdot$ Striatum $\cdot$ Striatopallidal $\cdot$ CAG repeat $\cdot$ tRFs $\cdot$ Polyglutamine disorders

Jordi Creus-Muncunill and Anna Guisado-Corcoll have contributed equally to this work.

Esther Pérez-Navarro

estherperez@ub.edu

$\triangle$ Eulàlia Martí

eulalia.marti@ub.edu

1 Departament de Biomedicina, Facultat de Medicina i Ciències de la Salut, Institut de Neurociències, Universitat de Barcelona, Casanova 143, Barcelona, Catalonia, Spain

2 Institut d'Investigacions Biomèdiques August Pi i Sunyer (IDIBAPS), Barcelona, Catalonia, Spain

3 Centro de Investigación Biomédica en Red sobre Enfermedades Neurodegenerativas (CIBERNED), Barcelona, Catalonia, Spain

4 Centre for Genomic Regulation (CRG), The Barcelona Institute for Science and Technology, Dr. Aiguader 88, Barcelona, Catalonia, Spain
5 Department of Biostatistics, Harvard T.H. Chan School of Public Health, Boston, MA, USA

6 Centro de Investigación Biomédica en Red sobre Epidemiología y Salud Pública (CIBERESP), Barcelona, Catalonia, Spain

7 Bellvitge Biomedical Research Institute (IDIBELL), L'Hospitalet de Llobregat, Barcelona, Catalonia, Spain

8 Department of Neurology, National Reference Center for CJD Surveillance, University Medical Center Göttingen, Göttingen, Germany

9 Present Address: Department of Neurology, Icahn School of Medicine at Mount Sinai, New York, NY 10029, USA 


\section{Introduction}

Huntington's disease (HD) is a polyglutamine (polyQ) disorder caused by a CAG trinucleotide expansion in the exon-1 of the Huntingtin (HTT) gene [23]. HTT with CAG repeats $>36$ cause a progressive loss of specific brain cells, with the medium-sized spiny neurons (MSNs) from the caudate nucleus and putamen (striatum in mouse) as the most affected type [21], leading to motor disturbances. Conventional wisdom has held that mutant huntingtin protein (mHTT) is the main causative effector in HD pathophysiology, through mechanisms including proteome disruption, transcriptional perturbations, and neurotransmitter release alterations, among others [37]. In addition, RNA gain-of-function has been identified as a relevant pathogenic mechanism in HD and other nucleotide repeat expansion disorders, such as myotonic dystrophy types 1 and 2, Fragile $X$ tremor ataxia syndrome, spinocerebellar ataxias 3, 8, 10, 12, 41, 36, Huntington's disease-like 2 , and C9 amyotrophic lateral sclerosis/frontotemporal dementia (for review, see [71]).

Others and we have previously shown that expanded CAG repeats assume a toxic gain-of-function at the mRNA level in HD through diverse mechanisms [4, 40, 77]. Expanded CAG repeats form semi-stable hairpins [35] that affect the subcellular localization of the mutant $H T T$ transcript and mediate the sequestration of muscleblind-like 1, SRSF6, U2AF65, and nucleolin [44]. As a result, defects in alternative splicing $[49,67]$ and altered levels of specific genes [74] are detected in HD brains. The detrimental effects of expanded CAG repeat RNAs are complemented by the neurotoxic activity of small CAG repeated RNAs (sCAG), of approximately 21 nucleotides in length. sCAG are processed from the mutant $H T T$ transcript by the endonuclease Dicer [34] and show damaging, gene-silencing activity [4, 34]. We previously showed that blockage of the CAG repeats partly ameliorates motor dysfunction in the R6/2 mouse model of $\mathrm{HD}$; but the specific contribution of sCAG or expanded CAG repeats (hairpin precursor) is not resolved. In addition, the pathogenic impact of other classes of bioactive small RNAs (sRNA) strongly altered in HD brains [45] is not clear. More recently, novel mechanisms beyond RNA gain-of-function or polyQ toxicity are emerging as relevant in HD field. Repeat associated non-ATG (RAN) translation results in the accumulation of toxic proteins in vulnerable regions of HD human brains [3]. In light of the potential coexistence of multiple neuropathological mechanisms in HD, here, we employed a number of strategies aimed at interrogating the deleterious role of sRNA species, disentangling RNA versus RAN or polyQ toxicity.

Herein, we injected sRNA purified from the putamen of non-affected individuals (CTL-sRNA-PT) and HD patients
(HD-sRNA-PT) into the striatum of wild-type (WT) mice. Then, we performed a comprehensive panel of behavioral, biochemical, and transcriptomic assays (see Fig. 1 for the experimental design). Importantly, we observed that HD-sRNA-PT induce motor deficits, accompanied by selective neuronal death and inflammation in the striatum, which are typical neuropathological features of HD human brains. By selectively blocking sCAG activity, we show that these species are partial contributors to the detrimental effects of HD-sRNA, expected from our previous observations [4, 61], but do not explain other important pathogenic outcomes. Moreover, we deeply characterized the sRNA composition to identify additional sRNA species involved in neuronal toxicity and observed that tRNA fragments (tRFs) are the most abundant representatives in HD putamen. Among these, we show that a highly abundant tRF derived from an Alanine tRNA compromises striatal neuronal viability in vitro, suggesting that diverse classes of sRNA may contribute to HD pathophysiology. Collectively, our data underlie the relevance of RNA detrimental mechanisms in HD and suggest that RNA species, other than $\mathrm{SCAG}$, should be a further priority in translational studies.

\section{Materials and methods}

\section{Mice}

For this study, adult male WT B6CBA mice were used. All mice were housed individually, and data were recorded for analysis by microchip mouse number. Animals were housed with ad libitum access to water and food in a colony room maintained at $19-22{ }^{\circ} \mathrm{C}, 40-60 \%$ humidity and under a standard 12:12 h light/dark cycle. Mice weight was controlled throughout all the experiments.

\section{Post-mortem human brain tissue}

Frozen samples (putamen, frontal cortex, and cerebellum) from HD patients and non-affected individuals were supplied by the Neurological Tissue Bank of the Biobank-Hospital Clínic-Institut d'Investigacions Biomèdiques August Pi i Sunyer (IDIBAPS; Barcelona, Catalonia, Spain). Genotype, Vonsattel stage, and sex and age information are provided in Online Resource 1.

\section{RNA isolation and small RNA species isolation}

Both human and mouse dissected brain areas were placed in QIAzol solution (QIAGEN; 79306), followed by RNA extraction with the miRNeasy mini kit (QIAGEN; 217004) as indicated by the manufacturer. Determinations of RNA 
Fig. 1 Schematic diagram of the study design. Frozen brain tissues from HD patients, as well as age-matched unaffected individuals were used to purify small RNAs (sRNA). Purified sRNA were subjected to small RNA-sequencing. sRNA were injected into the striatum of wild-type mice using bilateral cannulae. Artificial cerebrospinal fluid (ACSF) was used as control. Injected mice were assessed for motor behavior. Brains were processed for histopathological evaluation and transcriptomic analysis

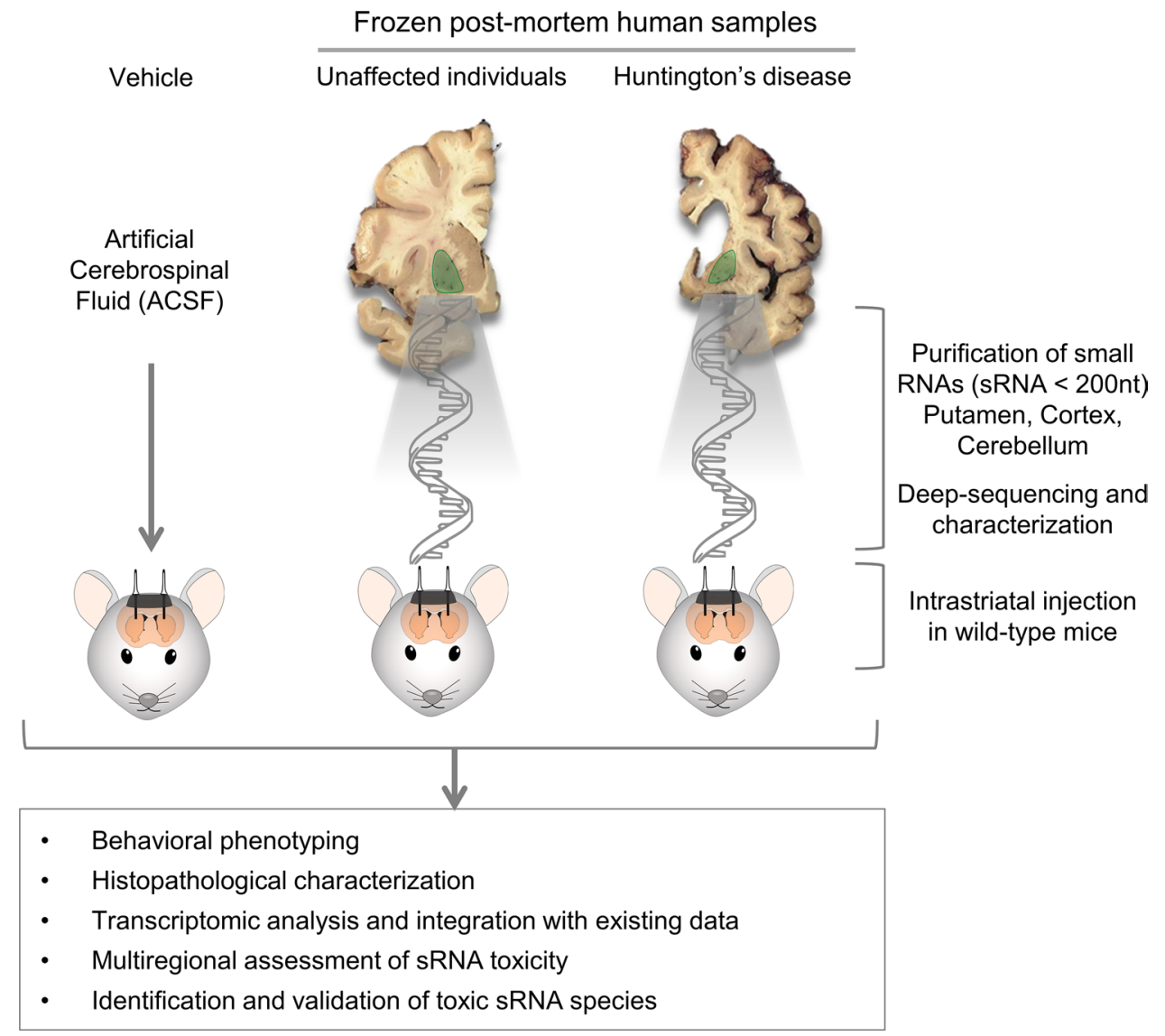

As (sRNA < 200nt) utamen, Cortex -sequencing and characterization

trastriatal injection in wild-type mice quality and quantity were made with a 2100 Bioanalyzer (Agilent Technologies) and an ND-1000 spectrophotometer (Thermo Fisher Scientific), respectively. All RNA samples showed an RNA integrity number (RIN) of 7 or higher. Small RNA (sRNA) fractions were purified from total RNA with the RNA Clean \& Concentrator-5 Kit (Zymo Research; $\mathrm{R} 1015)$ according to the manufacturer's instructions.

\section{Oligonucleotides}

The locked nucleic acid anti-sense oligonucleotides (LNAASO) complementary to the CAG repeat (LNA-CTG) consisted of a 21-nt length oligonucleotide, 5'-CTGCTGCTG CTGCTGCTGCTG-3', with an LNA located every third T nucleotide and a phosphorothioate-modified backbone [61]. LNA-CTG and the analogous control scrambled LNA-modified sequence (LNA-SCB), 5'-GTGTAACACGTCTATACG CCCA-3', were obtained from Exiqon. Human sRNA were co-injected with either LNA-CTG or LNA-SCB in a 1:2 quantity ratio.

Synthetic RNA oligonucleotides that mimic tRFs were purchased from Eurogentec (Liège, Belgium). The sequences of the RNAs used are: tRF-Ala-SS 5'-GGGGGU GUAGCUCAGUGGUAGAGCGCGUGC-3', tRF-Ala-AS 5'-GCACGCGCUCUACCACUGAGCUACACCCCC-3',
tRF-Gly-SS 5'- GCGCCGCUGGUGUAGUGGUAUCAU GCAAGAU-3', tRF-Gly-AS 5'-AUCUUGCAUGAUACC ACUACACCAGCGGCGC-3', tRF-Val-SS 5'-GGUUCC AUAGUGUAGUGGUUAUCACGUCUGCUUU-3' and tRF-Val-AS 5'-AAAGCAGACGUGAUAACCACUACA CUAUGGAACC-3'. All sequences present a 5' modification with a phosphate group.

\section{Intrastriatal injection}

Mice were anesthetized with isoflurane and placed into a stereotaxic apparatus in a flat skull position. During all procedures, body temperature was maintained constant using a heating pad. Stainless steel bilateral cannulas (26-gauge; Bilaney Consultants Ltd.) were implanted at the following coordinates: anteroposterior $(\mathrm{AP})+0.6 \mathrm{~mm}$, mediolateral $(\mathrm{ML}) \pm 2 \mathrm{~mm}$, and dorsoventral (DV) $-1 \mathrm{~mm}$ from bregma. Cannulas consist in a horizontal plate that joins two guide cannulas in which either dummy cannulas (without projection) or infusion cannulas (1 $\mathrm{mm}$ projection) can be introduced. Two screws were located into the skull to serve as anchors and the assemblage was fixed in place with dental cement. Two removable occluding dummy cannulas were inserted into the guide cannulas and were only removed prior to infusions. After surgery, all animals received a 
subcutaneous injection of meloxicam (Metacam, $0.2 \mathrm{ml}$ of $2 \mathrm{mg} / \mathrm{ml}$ ) to provide postoperative analgesia and were kept on a warm place until recovered from anesthesia.

Pools of sRNA samples obtained from equivalent amounts of $n=10-15$ patients per group were prepared (Online resource 1) to inject into the mice brain. Infusions were performed at $0.25 \mu \mathrm{l} / \mathrm{min}$ using an infusion pump. Bilateral infusions of $2 \mu \mathrm{l}$ of vehicle, CTL-sRNA-PT, HD-sRNA-PT, HD-sRNA-PT + LNA-SCB, HD-sRNA-PT + LNA-CTG, CTL-sRNA-PT + LNA-SCB, and CTL-sRNA-PT + LNACTG $(0.24 \mu \mathrm{g} / \mu \mathrm{l})$ were carried out. Another group of WT mice received similar intrastriatal injections of pure sCAG RNAs or a scrambled, control sequence (sSCB). WT mice were analogously injected with sRNA from the cortex (CTX) or cerebellum (CB) from non-affected individuals (CTL) and HD patients: CTL-sRNA-CTX, HD-sRNA-CTX, CTL-sRNA-CB, and HD-sRNA-CB. Artificial cerebrospinal fluid (ACSF) (in mM: $125 \mathrm{NaCl}, 2.5 \mathrm{KCl}, 1.2 \mathrm{NaH}_{2} \mathrm{PO}_{4}, 1.2$ $\mathrm{MgCl}_{2}, 2.4 \mathrm{CaCl}_{2}, 26 \mathrm{NaHCO}_{3}$, and 11 glucose; with $\mathrm{pH}$ adjusted to 7.2) was used as vehicle. Infusions were administered twice per week, for 2 weeks, and were preceded by a vehicle infusion (see experimental design in Fig. 2a). Infusion cannulas were left in place for $5 \mathrm{~min}$ after the injection to ensure a complete diffusion. Animals were sacrificed $48 \mathrm{~h}$ after the last infusion, and the cerebral hemispheres were processed for histology and protein or RNA extraction.

\section{Behavioral tests}

\section{Rotarod}

Motor coordination was evaluated on the rotarod apparatus at distinct rotations per minute $(\mathrm{rpm})$. Animals were trained at distinct constant speeds $(4,8,16$, and $24 \mathrm{rpm})$ for $60 \mathrm{~s}$ and the number of falls was recorded. No differences were detected between groups during this period. After training, animals were evaluated once a week at 16, 24 , and $32 \mathrm{rpm}$, and the latency to fall was registered. Performance was measured as the average latency to fall that animals achieved during 3 trials at each speed.

\section{Balance beam}

Motor coordination and balance were assessed by measuring the ability of mice to traverse a narrow beam as described in Ref. [9], with brief modifications. The beam consisted of a long steel cylinder $(50 \mathrm{~cm})$ with a $15 \mathrm{~mm}$ round diameter placed horizontally $40 \mathrm{~cm}$ above the floor and divided in $5 \mathrm{~cm}$ frames. Animals were allowed to walk for 2 min along the beam, and the number of slips, the total distance traveled, and the latency to cover 30 frames were measured.

\section{sRNA incorporation in striatal cells}

Purified small RNA fractions (8-10 ng) were labeled using the Label IT siRNA tracker intracellular localization kit Cy3 (Mirus; MIR7212) according to the manufacturer's instructions. Two microliters of Cy3-labeled small RNAs $(0.24 \mu \mathrm{g} /$ $\mu \mathrm{l})$ were unilaterally infused into the striatum of WT mice at $0.25 \mu \mathrm{l} / \mathrm{min}$ using an infusion pump. Animals were sacrificed $1 \mathrm{~h}$ after the infusion and the cerebral hemispheres were processed for histology.

\section{Small RNA-sequencing of human samples}

CTL-sRNA-PT, HD-sRNA-PT, CTL-sRNA-CTX, HD-sRNA-CTX, CTL-sRNA-CB, and HD-sRNA-CB pools $\left(200 \mathrm{ng}\right.$ ) were processed with NEBNext ${ }^{\circledR}$ Small RNA Library Prep Set for Illumina ${ }^{\circledR}$ (New England Biolabs; E7330S), size selection was performed by Novex ${ }^{\circledR}$ Pre-Cast 6\% TBE PAGE gel (Thermo Fisher Scientific; EC62652BOX) and the bands of interest cut as follows: (a) 145-160 bp band (corresponding to adapter-ligated constructs derived from the 21 and 30 nt RNA fragments, respectively), (b) 160-180 bp band, (c) 180-200 bp band, (d) 200-300 bp band, and (e) 300-500 bp. Paired-end sequencing $(2 \times 50$ cycles, CTL-sRNA-PT, HD-sRNA-PT) or singleend sequencing $(1 \times 50 \mathrm{nt}$, CTL-sRNA-CTX, HD-sRNACTX, CTL-sRNA-CB, HD-sRNA-CB) of indexed cDNA libraries were then carried out on a HiSeq 2500 machine (Illumina), generating at least 50 million reads per sample. Sequencing was performed using v4 SBS and Cluster Kits (Illumina; FC-401-4002). sRNA sequencing data are available at Gene Expression Omnibus with accession number GSE165667.

\section{Small RNA-sequencing of mice samples}

Total RNA (800 ng) was isolated from the striatum of mice injected with HD-sRNA-PT, CTL-sRNA-PT, or vehicle. DNased samples were processed with NEBNext ${ }^{\circledR}$ Small RNA Library Prep Set for Illumina ${ }^{\circledR}$ (New England Biolabs; E7330S), pooled and purified; size selection was performed by Novex ${ }^{\circledR}$ Pre-Cast $6 \%$ TBE PAGE gel (Thermo Fisher Scientific; EC62652BOX) and the band of interest (145-160 bp) excised from the gel. Single Read $50(1 \times 50$ cycles) sequencing of indexed cDNA libraries was then carried out on a HiSeq 2500 machine (Illumina), generating at least 50 million reads per sample. Sequencing was performed using v4 SBS and Cluster Kits (Illumina; FC-401-4002). 


\section{Bioinformatic analysis of sRNA data}

We used cutadapt [46] to remove $3^{\prime}$ adapter sequence from CTL-sRNA and HD-sRNA samples. Sequences that had the adapter were analyzed with bcbio-nextgen smallRNA-seq pipeline (https://www.bcbio-nextgen.readthedocs.io/en/lates $\mathrm{t} /, 1.0 .2 \mathrm{a} 0-\mathrm{a} 003647$ ) that uses a series of tools to characterize small RNA sequences (seqbuster [52], seqcluster [53], STAR [12], multiqc [14]). Sequences with no $3^{\prime}$ adapter were analyzed with bcbio-nextgen RNA-seq pipeline to measure the RNA quality (STAR [12], qualimap [17], multiqc [14]) and to quantify the gene expression of the samples (sailfish, [55]).

To detect human sRNA in the brain of mice injected with vehicle (ACSF), CTL-sRNA-PT, and HD-sRNA-PT, we used again bcbio-nextgen smallRNA-seq pipeline to map sequences in which the $3^{\prime}$ adapter was found. Sequences were mapped to the mouse (mm10) and human (hg19) genomes. Statistics of the bam file (aligned reads) were used to compare the amount of mapped sequences in each species.

\section{RNA-sequencing of mice samples}

Total RNA (500 ng) from the striatum of injected mice was extracted using the RNeasy Mini Kit (QIAGEN; 74104) and underwent DNase treatment to prevent genomic DNA contamination. RNA integrity was assessed using RNA 6000 Nano Chips on a 2100 Bioanalyzer (Agilent; 5067-1511). Samples with non-degraded RNA ( $R I N \geq 8.5)$ were used for sequencing. Preparation of indexed cDNA sequencing libraries was carried out using the TruSeq poly-A mRNA method (Illumina; 20020594). Briefly, poly-A mRNA transcripts were captured from total RNA using poly-T beads and cDNA was generated using random hexamer priming. Paired-end sequencing $(2 \times 50$ cycles $)$ of indexed cDNA libraries was then carried out on a HiSeq 2500 machine (Illumina), generating at least 50 million reads per sample. Sequencing was performed using v4 SBS and Cluster Kits (Illumina; FC-401-4002). RNA sequencing data are available at Gene Expression Omnibus with accession number GSE165667.

\section{RNA-sequencing data processing and analysis}

The quality of the fastq files was checked using the FastQC software (http://www.bioinformatics.babraham.ac.uk/proje cts/fastqc/). An estimation of ribosomal RNA in the raw data was obtained with riboPicker [69]. Reads were aligned with the STAR mapper (version 2.5.2a) to the GENCODE release M14 of the Mus musculus genome (GRHs38/hg38 assembly). A raw count of reads per gene was also obtained with STAR (-quantMode TranscriptomeSAM GeneCounts option; [12]). The R/Bioconductor [30] package DESeq2
[42] was used to assess differential expression between experimental groups (Wald statistical test + false discovery rate correction). Prior to processing the differential expression analysis, genes for which sum of raw counts across all samples were less than 2 were discarded. Gene ontology enrichments were performed using DAVID functional annotation tool [28, 29]. Cell-type enrichment analysis was calculated using Chi-square test. Cell-type specific genes were obtained from previously published data [47].

\section{RNA polyadenylation and polymerase chain reaction (PCR) amplification of SCAG and miR-16}

Total RNA was treated with TURBO DNA-free kit (Ambion; AM1907). In vitro polyadenylation reactions were carried out using $1 \mu \mathrm{g}$ of total RNA or $100 \mathrm{ng}$ of sRNA-enriched fraction and poly(A) polymerase (Ambion; AM2030) for $1 \mathrm{~h}$ at $37{ }^{\circ} \mathrm{C}$ in the presence of ATP $(1 \mathrm{mM})$. Samples were then annealed with a poly-T-adapter primer (5'-CGAATT CTAGAGCTCGAGGCAGGCGACATGGCTGGCTAGTTA AGCTTGGTACCGAGCTCGGATCCACTAGTCCTTTTT TTTTTTTTTTTTTTTTTTTTAC-3') prior to RT reaction. A specific primer recognizing the adapter primer (CGAATT CTAGAGCTCGAGGCAGG) was used in combination with either sCAG6 (CAGCAGCAGCAGCAGCAG) or miR-16 (ACACTCCAGCTGGGTAGCAGCACGTAAAT) specific primers to allow the amplification of specific RT-PCR products which were resolved on an acrylamide gel [61].

\section{Quantitative PCR (qPCR)}

Total RNA from mouse striatum was treated with TURBO DNAse I (Ambion, Thermo Fisher Scientific; AM1907) and retro transcribed using Transcriptor First Strand cDNA Synthesis Kit (Roche; 04897030001) following the manufacturer's instructions. cDNA was mixed with LightCycler 480 SYBR Green I (Roche, 04707516001) and amplification performed in a LightCycler 480 Real-Time PCR System (Roche; 05015278001). For each gene, samples were analyzed in the same RT-PCR experiment and run in quadruplicate.

For proinflammatory and immune-related genes qPCR determinations were carried out in 384-well optical plates using technical duplicates per cDNA sample in an ABI Prism 7900 Sequence Detection system (Applied Biosystems, Life Technologies) following supplier's parameters: $50{ }^{\circ} \mathrm{C}$ for $2 \mathrm{~min}, 95^{\circ} \mathrm{C}$ for $10 \mathrm{~min}$, and 40 cycles of $95^{\circ} \mathrm{C}$ for $15 \mathrm{~s}$ and $60^{\circ} \mathrm{C}$ for $1 \mathrm{~min}$. A total volume of $10 \mu \mathrm{l}$ TaqMan reaction contained $4.5 \mu \mathrm{l}$ cDNA, $0.5 \mu \mathrm{l}$ 20XTaqMan Gene Expression Assays, and $5 \mu \mathrm{l}$ of $2 \mathrm{X}$ TaqMan Universal PCR Master Mix (Applied Biosystems) per each sample. Data were processed by the Sequence Detection Software (SDS version 2.2.2, Applied Biosystems). 
Relative quantification (RQ) was calculated with the $2 \Delta \Delta \mathrm{Ct}$ method [41] using Hprt or actin as reference gene (specified in the corresponding figure legend). RQ was calculated to compare all expression values normalized to the reference gene in animals injected with vehicle, HD-sRNAPT or CTL-sRNA-PT, HD-sRNA-PT + LNA-SCB, and HD-sRNA-PT + LNA-CTG. Primers are listed in Online Resource 2.

\section{Protein extraction and western blot analyses}

Protein extraction and western blot (WB) were performed as described elsewhere [64]. The following primary antibodies were used at indicated dilutions: DARPP-32 (clone 15; 1:1000; Bd Bioscience, 611520), STEP (clone 23E5; 1:2000; Santa Cruz Biotechnology; sc-23892), PSD-95 (7E3-1B8; 1:1000; Thermo Scientific; MA1-046), PHLPP1 (1:1000; Cayman Chemical; 10007191), DRD2 (1:1000; Merck Millipore; AB5084P), ENK (1:1000; Abcam; ab85798), and DRD1 (H-109; 1:1000; Santa Cruz Biotechnology; sc-14001). All of them were incubated overnight at $4{ }^{\circ} \mathrm{C}$. Mouse monoclonal antibody against GAPDH (1:2000; Merck Millipore; AB2302) was used as loading control. After incubation with primary antibody, membranes were washed with TBS-T, incubated for $1 \mathrm{~h}$ at room temperature (RT) with the appropriated horseradish peroxidase-conjugated secondary antibody (1:2000; Promega; W4011 and W4021), and washed again with TBS-T. Immunoreactive bands were visualized using the Western Blotting Luminol Reagent (Santa Cruz Biotechnology; sc-2048) and finally quantified using a computer-assisted densitometer (Gel-Pro Analyzer, version 4, Media Cybernetics).

\section{Primary neuronal cultures and cell viability assay}

Brains from mouse E17.5 embryos were obtained and placed in Neurobasal medium (Gibco; 21103-049). Striata were dissected and gently dissociated with a fire-polished glass Pasteur pipette. Neurons were cultured in 96-well plates, pre-coated with $0.1 \mathrm{mg} / \mathrm{ml}$ poly-D-lysine (Sigma; P0899). Cultures were maintained at $37{ }^{\circ} \mathrm{C}$ in a humidified atmosphere containing $5 \% \mathrm{CO}_{2}$ in Neurobasal medium supplemented with B27 (Gibco; 17504-044) and Glutamax (Gibco; 35050-038) until 7 days in vitro (DIV). To determine cell viability, CellTiter $96^{\circledR}$ AQueous One Solution Cell Proliferation Assay (MTS) (Promega; G3580) was used. Primary striatal cultures were treated with $200 \mathrm{ng}$ of different tRFs and incubated for $1 \mathrm{~h}$. Then, MTS solution was added to the media and absorbance was measured at $490 \mathrm{~nm}$. Hydrogen peroxide (Sigma; H1009; $200 \mu \mathrm{M}$ ) was used as positive control.

\section{Immunofluorescence and immunohistochemistry}

Cerebral hemispheres were fixed by immersion in $4 \%$ paraformaldehyde (PFA) solution in $0.1 \mathrm{M}$ sodium phosphate for 3 days. Tissues were then cryoprotected by immersion for $24 \mathrm{~h}$ in $15 \%$ and $30 \%$ sucrose in PBS with $0.02 \%$ sodium azide. Samples were cut into $30 \mu \mathrm{m}$ serial coronal sections on a cryostat after freezing them in dry ice-cooled 2-methylbutane. Immunofluorescence was performed as described elsewhere [62]. Briefly, free-floating brain sections were incubated overnight at $4{ }^{\circ} \mathrm{C}$ with rabbit anti-cleaved caspase-3 (Asp175; 1:500; Cell Signaling; 9661), rabbit antineuropeptide Y (1:500; Abcam; ab30914), goat anti-choline acetyltransferase (1:500; Merck Millipore; AB144P), rabbit anti-parvalbumin (1:500; Swant; PV27), rat anti-ctip2 (1:400; Abcam; ab18465), rabbit anti-Iba1 (1:500; Wako; 019-19741), rabbit anti-Sox9 (1:500; Merck; AB5535), or rabbit anti-Olig2 (1:200; Millipore; ab9610). After incubation with primary antibodies, slices were washed and then incubated for $2 \mathrm{~h}$ at RT with the corresponding fluorescent secondary antibodies: AlexaFluor 555 goat anti-rabbit (1:200; Jackson ImmunoResearch Laboratories; 111-165-003), AlexaFluor 488 donkey anti-rabbit (1:200; Jackson ImmunoResearch Laboratories; 711-545-152), AlexaFluor 488 donkey anti-goat (1:200; Jackson ImmunoResearch Laboratories; 705-545-003), and AlexaFluor 488 goat anti-rat (1:200; Jackson ImmunoResearch Laboratories; 112-545-003. As negative controls, slices were incubated in the absence of primary antibodies and no signal was detected. For nuclear staining, tissue sections were placed on coverslips using DAPI Fluoromount-G (Southern Biotech; 0100-20) as mounting medium. Representative images were acquired using a Zeiss LSM880 confocal microscope or an Olympus BX60 epifluorescence microscope (Olympus, Tokyo, Japan) equipped with an Orca-ER cooled CCD camera (Hamamatsu Photonics, Hamamatsu, Japan) and analyzed using CellProfiler Analyst software [32].

For diaminobenzidine (DAB) immunohistochemistry, brain slices were incubated overnight at $4{ }^{\circ} \mathrm{C}$ with mouse anti-DARPP-32 (1:1000; BD Bioscience; 611520). After primary incubation, an ABC Peroxidase Standard Staining Kit (Thermo Fisher Scientific; 32020) was used to obtain stained cells by incubation of brain sections with 3,3'-DAB. Stained cells were visualized using light microscope and morphometric analyses were performed using the ComputerAssisted Stereology Toolbox (CAST) software (Olympus). The stereological evaluation was performed using a morphometric system consisting of an Olympus BX51 microscope (Olympus, Tokyo, Japan) equipped with a motorized stage. The mean number of stained cells per cubic millimeter of tissue was determined for each animal. A minimum of four fields from six to eight different coronal sections per animal were counted. Sections were spaced at $240 \mu \mathrm{m}$ apart comprising the dorsal striatum. 
For Nissl staining, sections were placed in pre-treated slides and immersed in $0.1 \%$ cresyl violet (Fluka; 61123) for $45 \mathrm{~min}$. After dehydration in ethanol solutions of increasing concentrations (one step in ethanol $70 \%$, one step in ethanol $90 \%$, and two steps in ethanol 100\%; 5 min each step) and two steps in xylol, slides were fitted with coverslips and examined with a light microscope. The number of stained neurons was identified using morphological criteria $[18,19]$ and counted in four fields of the dorsal striatum from three different coronal sections per animal. All images were processed with Fiji/ImageJ free software.

\section{Statistics}

All data are expressed as the mean \pm SEM. Statistical analyses were performed using either ANOVA test or linear mixed-effects model (LMM) when technical replicates were taken into account [70], followed by Bonferroni's post hoc test as appropriate and indicated in figure legends. A 95\% confidence interval was used and values of $p<0.05$ were accepted as denoting statistical significance. Results were quantified and represented graphically using GraphPad Software (Inc., CA, USA).

\section{Study approval}

Human brain samples from HD patients and non-affected individuals were supplied by the Neurological Tissue Bank of the Biobank-Hospital Clínic-Institut d'Investigacions Biomèdiques August Pi i Sunyer (IDIBAPS; Barcelona, Catalonia) following the guidelines and approval of the local ethics committee (Hospital Clínic of Barcelona's Clinical Research Ethics Committee; HCB/2015/0088). Informed consent was obtained from all subjects under study. Regarding mice, all procedures were performed in compliance with the NIH Guide for the Care and Use of Laboratory Animals, and approved by the local animal care committee of Universitat de Barcelona following European (2010/63/UE) and Spanish (RD53/2013) regulations for the care and use of laboratory animals.

\section{Results}

\section{Intrastriatal injection of sRNA derived from the putamen of HD patients alters motor function and recapitulates HD molecular abnormalities in wild-type mice}

To investigate whether sRNA generated in the brain of HD patients could play a neurotoxic role in vivo, we developed a mouse model whereby sRNA purified from human brain tissue were intrastriatally injected in WT mice (Fig. 1). We obtained a fraction enriched in sRNA from the putamen of control individuals (CTL-sRNA-PT) and HD patients (HD-sRNA-PT) (Online Resource 1). Equivalent amounts of total RNA were mixed to obtain a representative pool of CTL-sRNA-PT $(n=10-14)$ and HD-sRNA-PT $(n=11-14)$. Then, sRNA $(<200 \mathrm{nt})$ were purified from each pool using a size exclusion column. RNA electrophoresis confirmed that purified sRNA pools were smaller than $200 \mathrm{nt}$ in length and the corresponding electropherogram showed that the most concentrated fraction corresponded to fragments of less than $40 \mathrm{nt}$ in length (Online resource 3 ). The most characteristic symptom in HD patients is the alteration of motor coordination, which is associated with the dysfunction/loss of striatal MSNs [76]. To study whether HD-sRNA-PT compromise motor behavior, vehicle, CTL-sRNA-PT, or HD-sRNA-PT were bilaterally injected into the striatum of WT mice at 4 different days (see Fig. 2a) and their motor performance examined using the rotarod and balance beam tests (Fig. 2b, c). HD-sRNAPT-injected mice showed significant motor alterations in both paradigms in comparison with CTL-sRNA-PT- or vehicle-injected mice. Motor perturbations in the rotarod were already detected following the two first injections of HD-sRNA-PT and worsened with the two subsequent injections (Fig. 2b). In the balance beam test, HD-sRNAPT-injected mice showed alterations in the number of slips per frame (Fig. 2c). Remarkably, the performance of CTLsRNA-PT-injected mice did not differ from mice injected with vehicle, indicating that CTL-sRNA-PT do not induce any negative effect on motor function.

To confirm the presence of human sRNA in the injected striata, RNA-sequencing analysis was conducted $48 \mathrm{~h}$ after the last infusion in the three experimental groups of mice. The proportion of reads mapping onto the human genome in mice injected with human sRNA (CTL-sRNA-PT or HD-sRNA-PT) was 6-10\% higher than in vehicle-injected mice (Online Resource 4), suggesting that human-specific sRNA were present in mice striatum during the evaluation of motor behavior. We also examined the type of cells incorporating human sRNA through injection of fluorescently tagged human CTL-sRNA into the striatum of WT mice and evaluation of their localization $1 \mathrm{~h}$ after the infusion. Interestingly, we observed that sRNA species colocalized with Ctip2-(MSNs marker) and Iba1(microglial marker), but not Sox9-(astrocytes marker) or Olig2-(oligodendrocyte marker) positive cells (Online Resource 5), and were preferentially localized in the nucleus. These results indicate that exogenous sRNA species are preferentially incorporated and integrated by striatal neurons and microglia, at least at $1 \mathrm{~h}$ post-injection.

Next, we examined if motor dysfunction induced by HD-sRNA-PT was paralleled by perturbations in the 
o Vehicle $\square$ CTL-sRNA-PT $\square$ HD-sRNA-PT

a

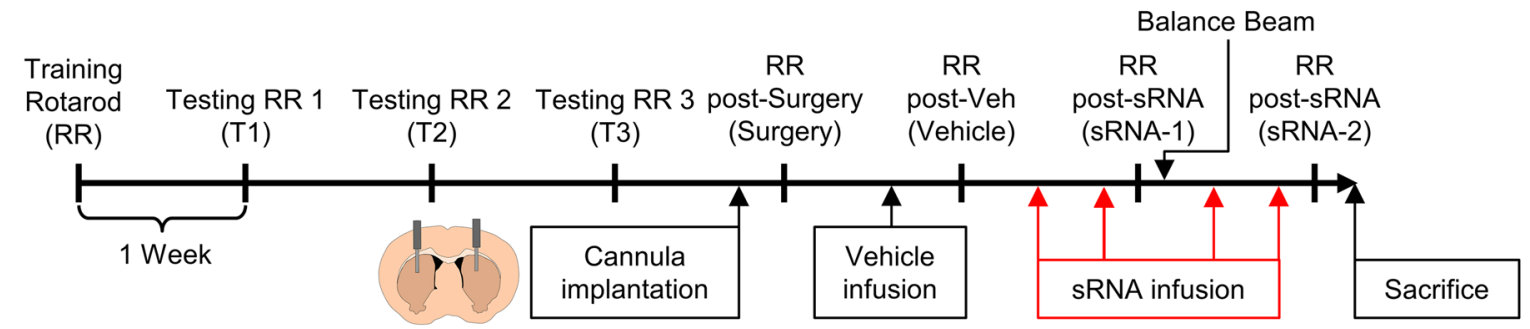

b
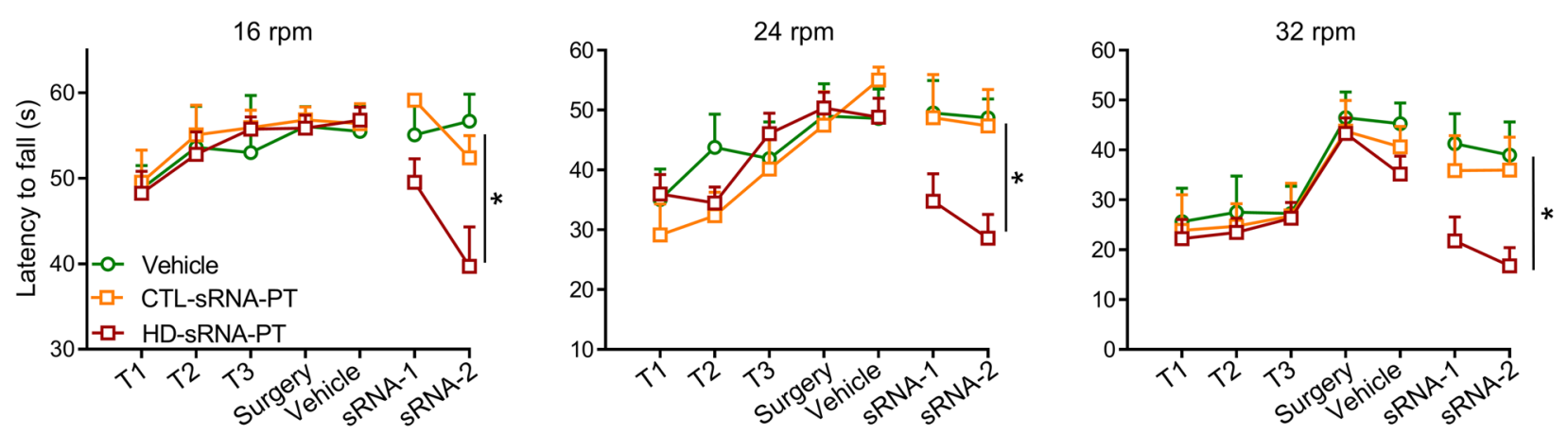

C
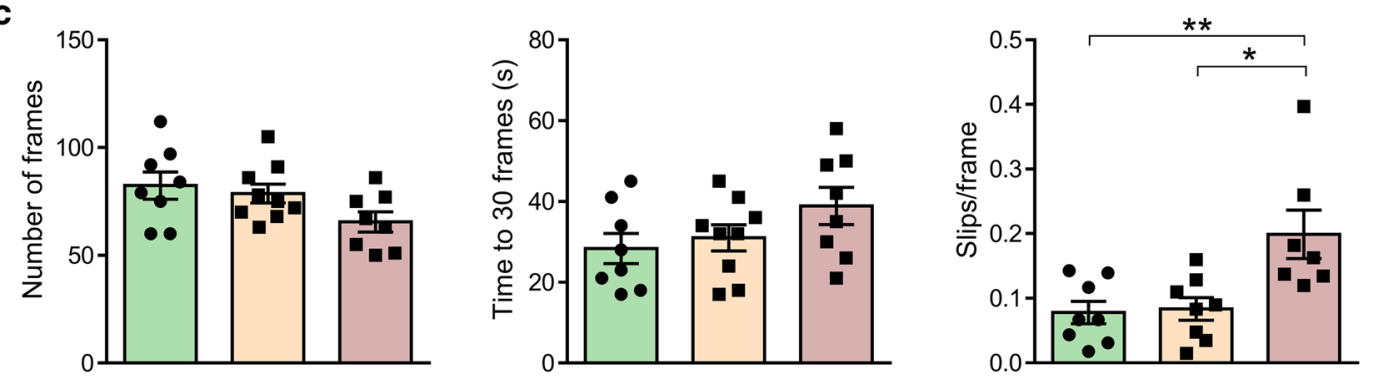

d
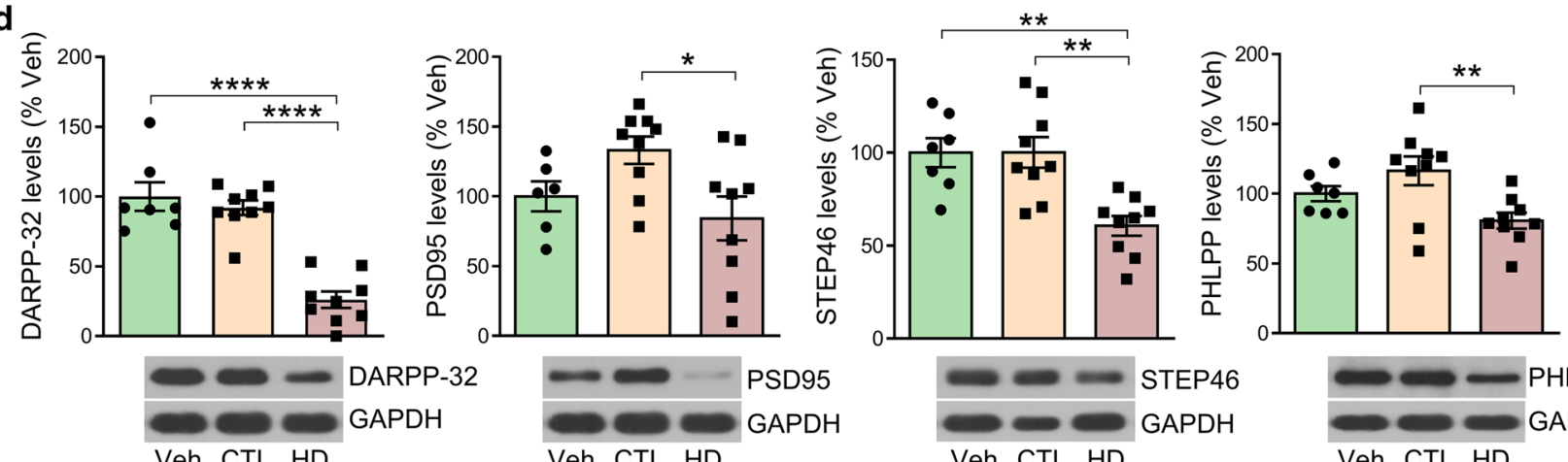

Veh CTL HD
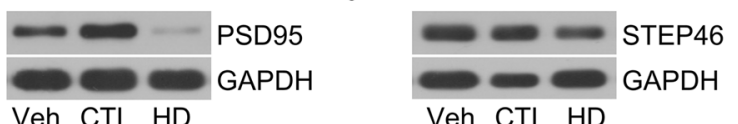

$\longrightarrow$ PHLPP GAPDH Veh CTL HD
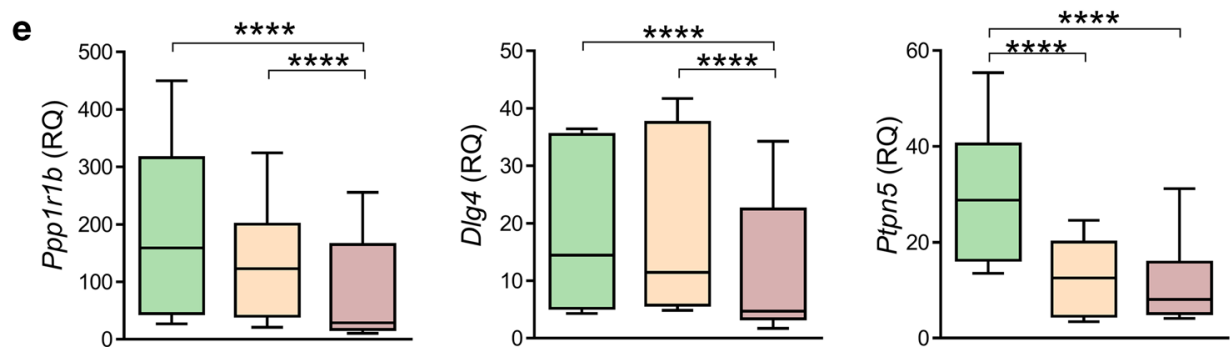
4Fig. 2 Intrastriatal injection of HD-sRNA-PT induce motor alterations in wild-type mice and alters the levels of several striatal HDrelated proteins. a Experimental procedure of intrastriatal infusions of vehicle or sRNA (CTL-sRNA-PT or HD-sRNA-PT) and motor coordination evaluation. $\mathbf{b}$ Motor performance in the rotarod test performed at 16, 24, and $32 \mathrm{rpm}$. Vehicle $n=8$; CTL-sRNA-PT $n=16$; HD-sRNA-PT $n=16$. c Motor coordination in the balance beam test performed $48 \mathrm{~h}$ after the second infusion of vehicle or sRNA. d Protein levels of DARPP-32, PSD-95, STEP46, and PHLPP were analyzed in striatal lysates by Western blot. GAPDH was used as a loading control. Representative immunoblots are shown. Vehicle $n=9$; CTL-sRNA-PT $n=9$; HD-sRNA-PT $n=9$. e Expression levels of DARPP-32 (Ppp1r1b), PSD-95 (Dlg4), and STEP (Ptpn5) determined by qPCR. Quantification was normalized to Hprt as a reference gene. Vehicle $n=6-8$; CTL-sRNA-PT $n=8$; HD-sRNA-PT $n=8$. In $\mathbf{c}-\mathbf{e}$, each point represents data from an individual mouse. All behavioral and biochemical data are shown as mean \pm SEM. $* * * * P<0.0001, * * P<0.01, * P<0.05$ versus all other groups; calculated by two-way ANOVA in $\mathbf{b}$; by one-way ANOVA in $\mathbf{c}, \mathbf{d}$ and using a linear mixed-effects model in e. In all cases, Bonferroni was used as post hoc test

expression of neuronal markers characteristically decreased in the striatum of HD: DARPP-32 [6, 10], Post-Synaptic Density-95 (PSD-95; [15, 73]), STriatal-Enriched protein tyrosine Phosphatase 46 (STEP46; [11, 43, 64]), and PH domain Leucine-rich repeat Protein Phosphatase 1 (PHLPP1; [61, 63]). Both protein (Fig. 2d) and mRNA (Fig. 2e) levels of DARPP-32 and PSD-95 were significantly decreased in the striatum of HD-sRNA-PT-injected mice compared with vehicle- or CTL-sRNA-PT-injected mice. Yet, while STEP46 protein was downregulated by HD-sRNA-PT, the corresponding mRNA was reduced in both CTL- and HD-sRNA-PT-injected mice.

The reason why certain HD brain regions are more vulnerable is still poorly understood. To elucidate if detrimental HD-sRNA are preferentially produced in vulnerable regions or analogously generated in less-affected areas, we sought to analyze whether HD-sRNA from the motor cortex (as affected) and the cerebellum (as less-affected) brain regions [60] could differently compromise motor function in WT mice. We injected CTL-sRNA and HD-sRNA, along with vehicle, obtained from the human cortex (CTL- or HD-sRNA-CTX) and cerebellum (CTL- or HD-sRNA-CB) following the same experimental approach described in Fig. 2. Mice injected with HD-sRNA-CTX showed motor deficits similar to mice injected with HD-sRNA-PT in contrast to mice injected with HD-sRNA-CB, which displayed motor deficits only at the most demanding speeds (Online Resource 6). In agreement with the results shown in Fig. 2, CTL-sRNA did not induce motor abnormalities in any paradigm. Altogether these data suggest that intrinsic biochemical properties of the most affected areas may underlie the biogenesis of detrimental sRNA, contributing to HD-associated profiles of regional vulnerability.

\section{HD-sRNA-PT induce selective gene expression alterations concordant with an HD-associated transcriptional signature}

To identify pathways underlying the detrimental effects of HD-sRNA-PT, we used RNA-sequencing to analyze gene expression patterns in the striatum of vehicle-, CTL-sRNAPT-, and HD-sRNA-PT-injected mice 2 days after the last injection. We detected a total of 1846 upregulated genes and 1358 downregulated genes in HD-sRNA-PT- versus vehicleinjected mice ( $n=8$ animals per group, Fold change $>1.5$ or $<1.5$, adjusted $P<0.05$; Fig. 3a; Online Resource 7) that were not significantly dysregulated when comparing CTL-sRNA-PT- versus vehicle-injected mice ( $n=8$ animals per group, adjusted $P>0.05$; Fig. 3b). Functional annotation of these differentially expressed genes (DEGs) revealed that downregulated genes were significantly enriched in neuronal pathways (Fig. 3c, Online Resource 8), whereas upregulated genes were enriched in immune system and inflammatory responses (Fig. 3d, Online Resource 9), according to the DAVID functional annotation tool [28, 29]. The comparison between CTL-sRNA-PT and HD-sRNA-PT experimental groups only revealed DEGs (adjusted $P<0.05$ ) enriched in the immune response, indicating that immune genes are the more consistently altered. We validated through qPCR the increase of inflammatory and immune-related genes such as Clcx2, Il1b, Ptgs2, and Tnf in HD-sRNA-PT-injected mice (Online Resource 10). Transcriptomic analysis confirmed the downregulation of the mRNA corresponding to Ppplr1b, Dlg4, and Ptpn5, as shown in Fig. 2e.

To better understand whether specific cell types were affected, we analyzed the overlap between the HD-sRNAPT DEGs and the genes identified as cell-type specific in a previous study [47]. Downregulated genes in HD-sRNAPT-injected striatum were enriched in neuronal-specific transcripts (Fig. 3e) especially in specific genes from MSNs expressing Dopamine Receptor D2 (DRD2-MSNs or indirect pathway; $P$ value $=2.2 \mathrm{e}-15$; Chi-square test) (Fig. 3e). Additionally, the majority of the upregulated genes in HD-sRNA-PT-injected striatum corresponded to glial-specific genes, especially enriched in microglial cells ( $p$ value $<2 \mathrm{e}-20$; Chi-square test) (Fig. 3f, Online Resource 11). These results suggest that HD-sRNA-PT contribute to specific neuronal subpopulation loss and microgliosis, both characteristic of HD patients' putamen [21, 66]. Subsequently, to investigate how HD-sRNA-PT signature resembles the HD-associated transcriptome, we computed the overlap of our DEGs with existing mouse and human HD transcriptomic datasets. The expression pattern of most DEGs was analogous to that of the human HD putamen $[13,25]$ and the striatum of a knock in HD mouse model [38] (Fig. 3g, h). This suggests that human HD-sRNA-PT 
a

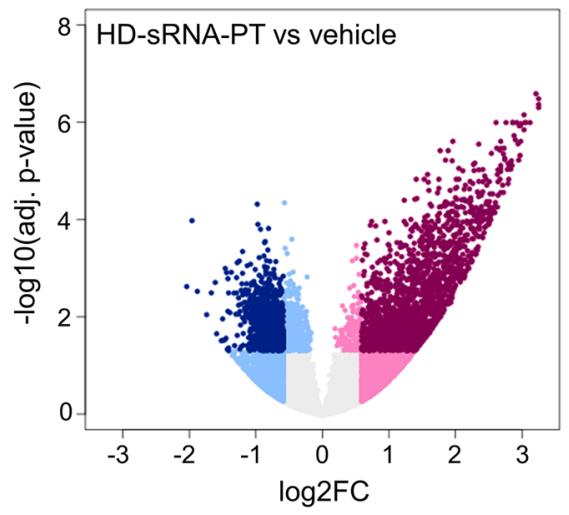

b

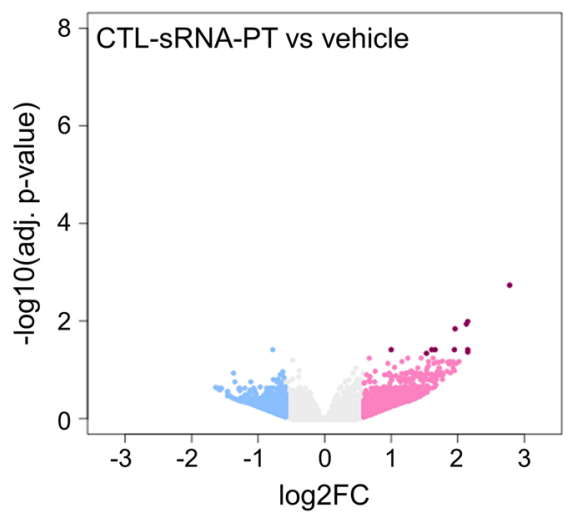

e

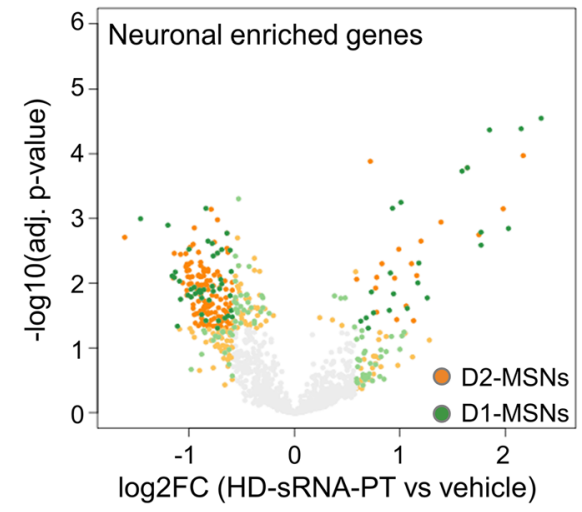

C

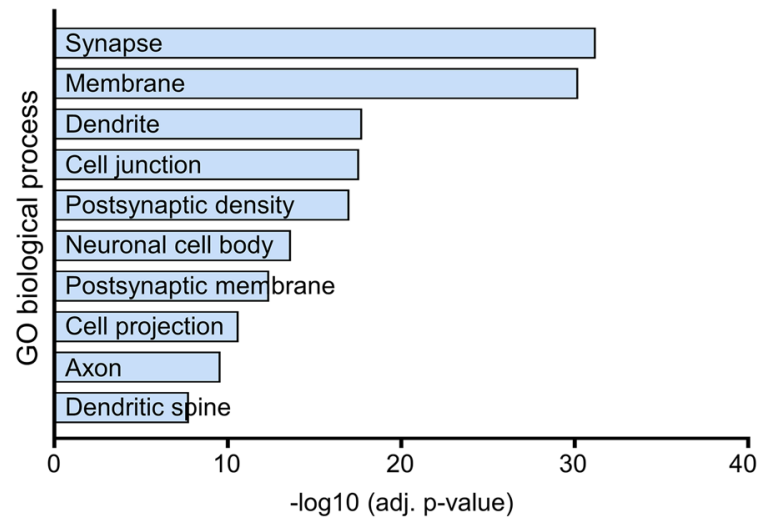

d

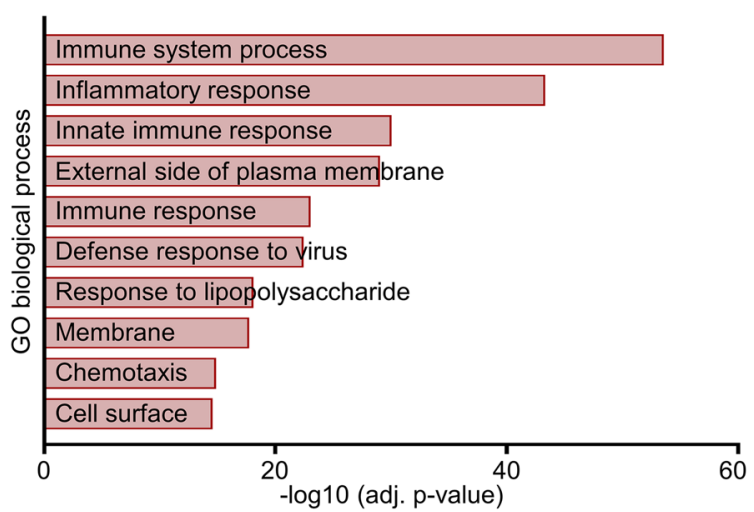

g

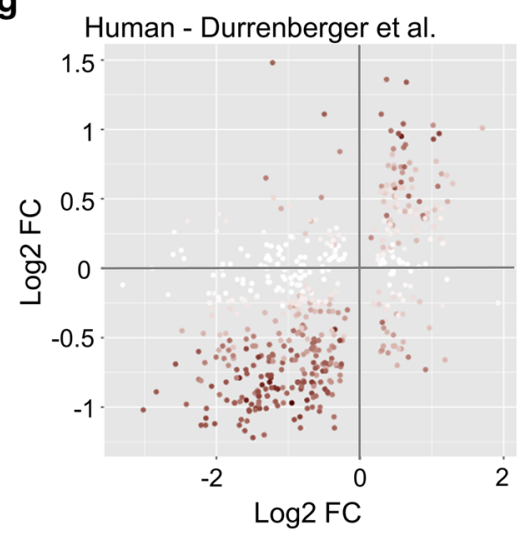

Human - Hodges et al.

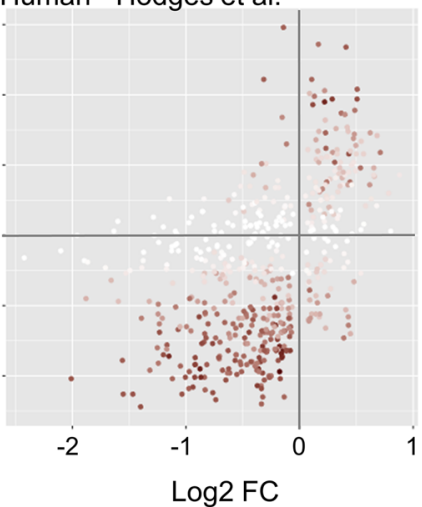

h

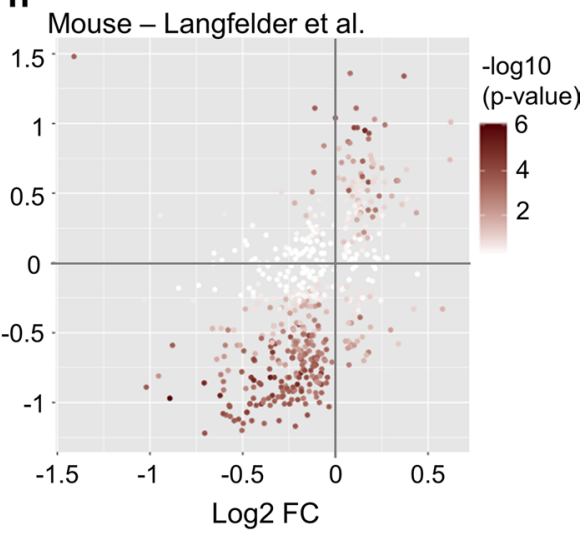


4Fig. 3 Intrastriatal injection of HD-sRNA-PT leads to transcriptional abnormalities resembling the HD-associated transcriptome. Volcano plot showing differentially expressed genes in a HD-sRNA-PT- versus vehicle-injected mice and b CTL-sRNA-PT versus vehicleinjected mice, $48 \mathrm{~h}$ after the last infusion. Dark purple dots represent significantly up-regulated genes (fold change $>1.5$, adjusted $P<0.05$ ) and dark blue dots represent significantly downregulated genes (fold change $<1.5$, adjusted $P<0.05$ ). GO biological processes associated with $\mathbf{c}$ down-regulated and $\mathbf{d}$ upregulated DEGs in HD-sRNA-PT versus vehicle-injected mice and not significantly altered in CTL-sRNAPT versus vehicle. e Volcano plot showing neuronal-enriched DEGs (fold change $>0.5$ or $<0.5$, adjusted $P<0.05$, dark color). Genes belonging to D1-MSNs are colored in green and genes belonging to D2-MSNs are colored in orange. f Volcano plot showing glialenriched DEGs (fold change $>0.5$ or $<0.5$; adjusted $P<0.05$, dark color). Genes belonging to astroglia are colored in orange and genes belonging to microglia are colored in blue. $\mathrm{g}$ Correlation between fold changes from HD-sRNA-PT versus vehicle-injected mice $(Y$ axis) and two independent HD human transcriptomic datasets. h Correlation between fold changes from HD-sRNA-PT versus vehicleinjected and mouse transcriptomic dataset

induce neuronal dysfunction and transcriptional perturbations resembling those taking place in HD.

\section{HD-sRNA-PT induces specific neuronal degeneration}

Specific loss of striatal MSNs is a major hallmark in HD but poorly recapitulated in mouse models [16]. To explore whether HD-sRNA-PT could compromise cell viability, we counted MSNs, using DARPP-32 immunohistochemistry. A reduction in the number of Nissl+ and DARPP-32+ cells was found in the striatum of HD-sRNA-PT-injected mice in comparison to vehicle- and CTL-sRNA-PT-injected animals (Fig. 4a, b), which was paralleled by a significant increase of cleaved caspase-3-positive cells (Fig. 4c). Furthermore, HD-sRNA-PT induced a significant decrease in DRD2 and enkephalin protein levels (Fig. 4d), suggesting a detrimental effect on the MSNs of the indirect pathway of the basal ganglia. These results are in line with the transcriptomic analysis showing decreased expression of D2-MSNs-specific genes (Fig. 3e). No obvious differences in the protein levels of Dopamine Receptor D1 (DRD1; Fig. 4d) and the number of neuropeptide Y-, choline acetyltransferase- or parvalbumin-positive neurons (Fig. 4e, Online Resource 12) were detected in the striatum of mice injected with HD-sRNAPT. Thus, the deleterious effect of HD-sRNA-PT may not be extensive to MSNs of the direct pathway and striatal interneurons, which are less-affected in HD [59]. These results are in line with the transcriptomic perturbations produced by HD-sRNA-PT (Fig. 3e), showing enrichment in D2-MSNs-specific genes, among the downregulated genes. Altogether, these results provide evidence that HD-sRNAPT compromise neuronal functionality and viability in vivo and suggest a deleterious effect on the most early affected striatal neuronal population in HD, i.e., D2-MSNs [21].
SCAG are partial contributors to the detrimental effects of HD-sRNA

We previously reported that $\mathrm{sCAG}$ species (21 nt) are increased in the HD human putamen and compromise cell viability in vitro [4]. Here, we detected SCAG in the purified pools of sRNA, with a moderate increase in the HD-sRNAPT pool in comparison with CTL-sRNA-PT pool (Online Resource 13), in accordance with the previous studies [4, 61]. As expected, the injected HD-sRNA-PT pool did not contain long-expanded CAG repeats within HTT-exon-1 that were detected in the total RNA fraction (Online Resource 13) [4]. Therefore, the possible detrimental effect of CAG repeats contained in the HD-sRNA-PT should be attributed to short CAG repeats such as $\mathrm{SCAG}$.

To determine to what extent $\mathrm{sCAG}$ were key species accounting for motor abnormalities and neuronal toxicity observed in HD-sRNA-PT-injected mice, we co-injected a locked nucleic acid anti-sense oligonucleotide targeting the CAG repeats (LNA-CTG; [61]) or an analogous control scrambled oligonucleotide (LNA-SCB), using the experimental approach described in Fig. 2a. Rotarod test evidenced a significant delay in the appearance of motor deficits in the HD-sRNA-PT + LNA-CTG-injected compared with the HD-sRNA-PT + LNA-SCB-injected mice at all speeds tested (Fig. 5a). Whereas HD-sRNA-PT infusion caused a rapid decrease in the latency to fall immediately after the first week of injections (Fig. 2a), HD-sRNA-PT + LNA-CTGinjected animals did not start presenting a significant motor deterioration until the second week of infusions (Fig. 5a). In agreement with these findings, in the balance beam test, performed after two injections of sRNA, only HD-sRNAPT + LNA-SCB-injected mice showed alterations (Fig. 5b).

Correlating with the delay in the appearance of motor dysfunction, our data suggest that the decrease in DARPP-32 protein levels and in the number of DARPP-32+cells was prevented in the striatum of HD-sRNA-PT + LNA-CTGinjected mice (Fig. 5c). However, the number of cleaved caspase-3 + cells was similar between HD-sRNA-PT + LNASCB- and HD-sRNA-PT + LNA-CTG-injected mice (Fig. 5d, e). In addition, LNA-CTG administration did not prevent the decrease in DRD2 protein levels (Fig. 5f; Online Resource 14), nor modified the levels of DRD1. Other markers strongly decreased by HD-sRNA-PT (Fig. 4d), such as Enkephalin, PSD-95, STEP46, or PHLPP1, showed a similar trend to decrease in HD-sRNA-PT + LNA-SCBinjected mice. However, their levels were not modified in HD-sRNA-PT + LNA-CTG-injected mice (Fig. 5f; Online Resource 14). Moreover, LNA-CTG did not induce significant effects in the levels of transcripts strongly altered in the HD-sRNA-injected mice (Online Resource 15), including Ppplrlb mRNA levels (Online Resource 16), suggesting a post-transcriptional mechanism of action of LNA-CTG. 
a

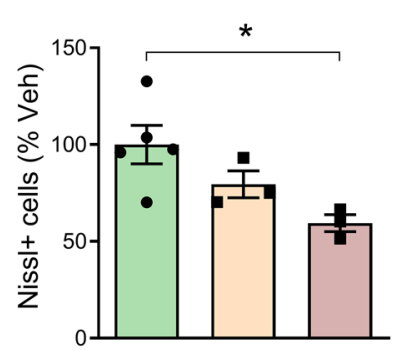

b

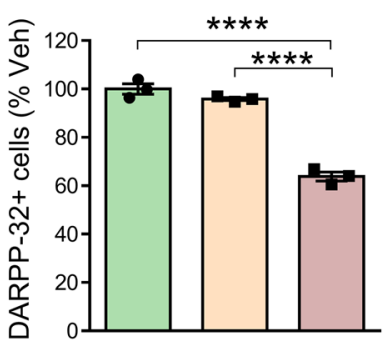

C

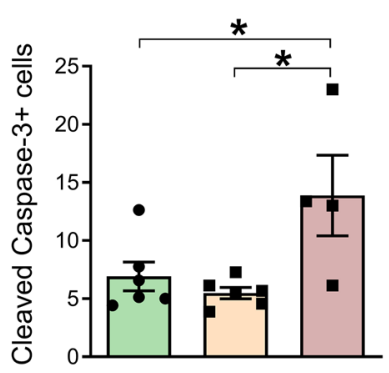

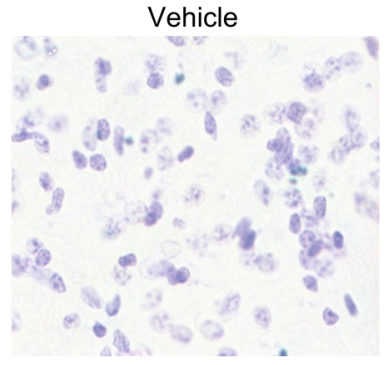

Vehicle

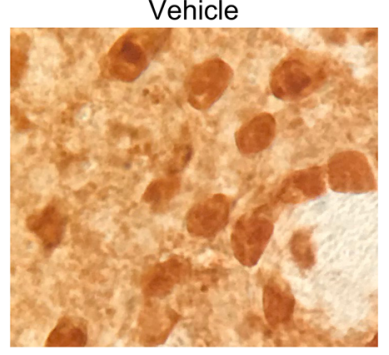

Vehicle
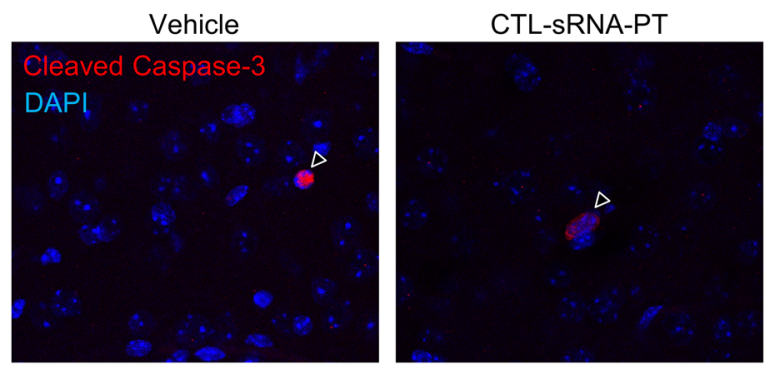

CTL-SRNA-PT
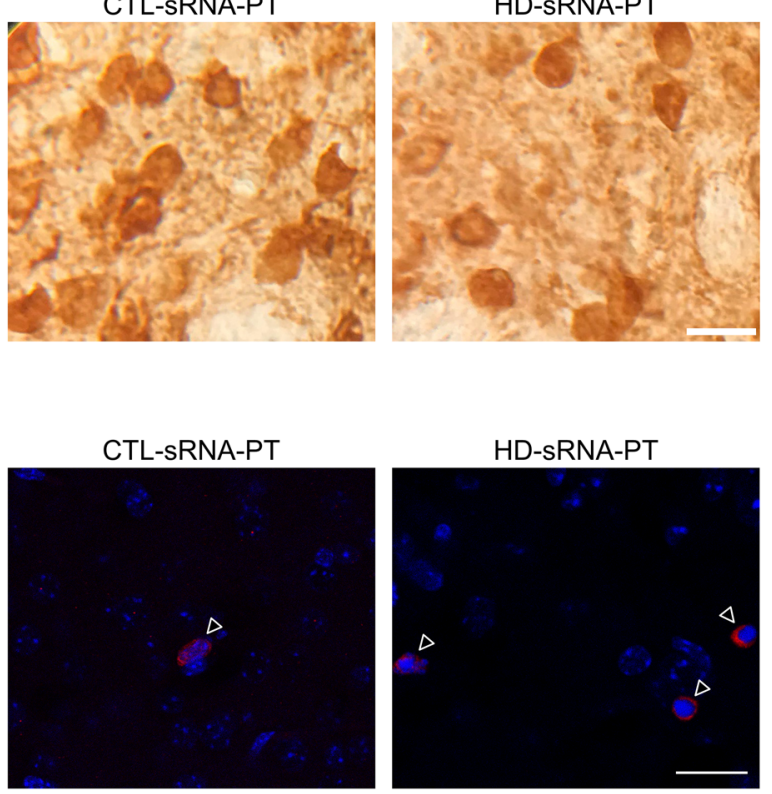

d
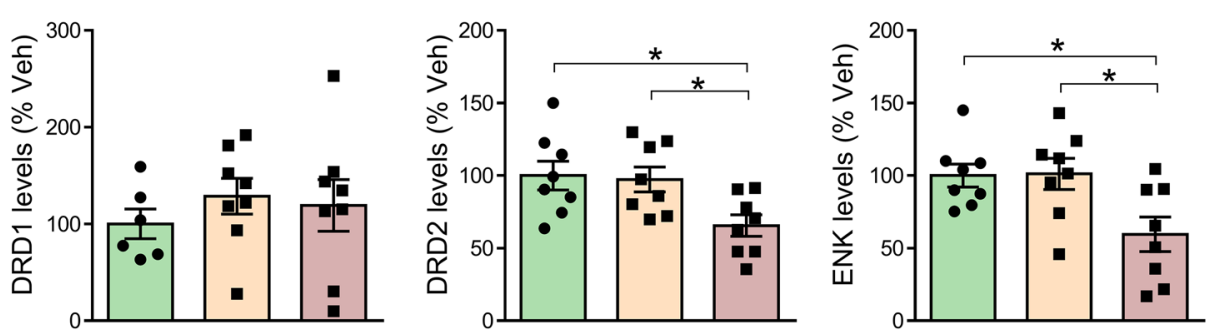

Veh CTL HD

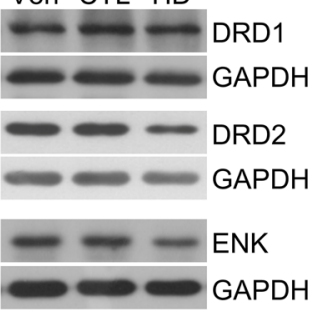

HD-sRNA-PT

i= $=$

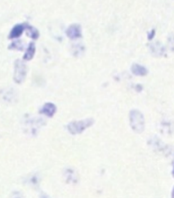

D-sRNA-PT

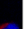

- e

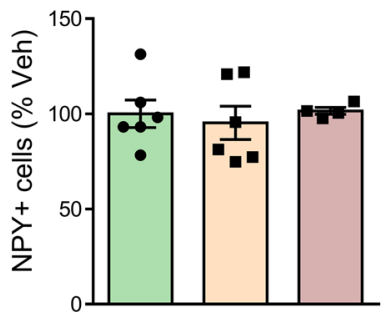

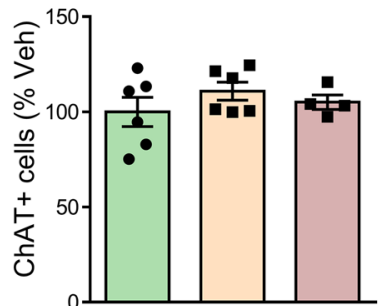

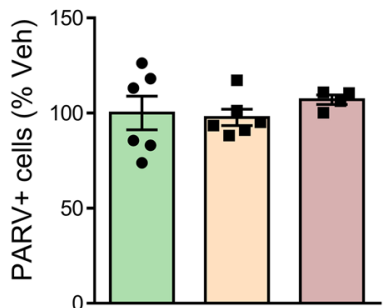


4Fig. 4 Intrastriatal injection of HD-sRNA-PT induces selective neuronal loss affecting the indirect pathway. Evaluation of a Nissl and b striatal DARPP-32-positive cells. Vehicle $n=5$; CTL-sRNA-PT $n=3$; HD-sRNA-PT $n=3$. Representative images are shown. Scale bar: $20 \mu \mathrm{m}$. c Graph shows the number of cleaved caspase-3-positive cells in the striatum in all the conditions analyzed. Vehicle $n=6$; CTL-sRNA-PT $n=6$; HD-sRNA-PT $n=4$. Representative images are shown. Scale bar: $25 \mu \mathrm{m}$. d Protein levels of DRD1, DRD2 and enkephalin were analyzed in striatal lysates by western blot. GAPDH was used as a loading control. Vehicle $n=6-8$; CTL-sRNAPT $n=8$; HD-sRNA-PT $n=8$. Representative immunoblots are shown. e Graphs showing the quantification of the number of NPY-, ChAT-, and PARV-positive cells in the striatum. Vehicle $n=6$; CTL-sRNA-PT $n=6$; HD-sRNA-PT $n=4$. Each point represents data from an individual mouse. All data are shown as mean \pm SEM. $* * * * P<0.0001, * P<0.05$ versus all other groups; calculated by oneway ANOVA with Bonferroni as post hoc test

These data indicate that sCAG do not explain important pathogenic effects of HD-sRNA-PT.

Analogous injection of LNA-CTG or LNA-SCB together with CTL-sRNA-PT did not result in significant alterations on the motor performance (Online Resource 17) nor changes in the levels of DARPP-32, PSD-95, STEP46, PHLPP1, DRD1, enkephalin, and DRD2 proteins, indicating that LNA-ASOs did not influence putative CTL-sRNA-PT effects (Online Resource 17).

The partial prevention of the detrimental effects caused by HD-sRNA-PT co-administrated with LNA-CTG catalyzed our efforts to further validate the deleterious effects of sCAG in vivo. We administered pure sCAG RNAs of 21 nt (sCAG_21) or a control scrambled RNA sequence (sSCB) in the striatum of WT mice, following the experimental approach depicted in Online Resource 18. Motor abnormalities were detected in both balance beam and rotarod tests, although only at the more demanding speed (32 rpm), in sCAG_21-injected compared to vehicle- and sSCB-injected mice (Online Resource 18). Alterations in motor coordination were accompanied by decreased DARPP-32 protein levels in the striatum of animals receiving sCAG_21 compared with vehicle- or sSCB-injected mice (Online Resource 18). However, the number of striatal MSNs remained unaltered (Online Resource 19), and accordingly, apoptotic cell death was not detected (Online Resource 19). The fact that the number of striatal MSNs remains unaltered in SCAG injected mice suggests that the detrimental activity of sCAG of $21 \mathrm{nt}$ is complemented by short RNA species with CAG repeats $(<40)$ that have been revealed in the HD human brain [4] and are likely targeted by LNA-CTG. In addition, PSD-95, STEP46, PHLPP1, DRD1, enkephalin, and DRD2 protein levels were not significantly altered (Online Resource 19). Overall, these data suggest that sRNA containing CAG repeats partially contribute to the neuronal dysfunction underlying motor deficits. However, specific neuropathogenic readouts are complemented by other sRNA species within the HD-sRNA-PT pool.

\section{Identification of potentially toxic HD-sRNA species}

To identify sRNA candidates for HD-sRNA-PT neurotoxicity, CTL-sRNA-PT and HD-sRNA-PT were characterized using deep sequencing. The electropherogram of the sRNA $(<200 \mathrm{nt})$ showed that the most abundant sRNA were less than $40 \mathrm{nt}$ in length. Bioinformatic analysis revealed that the majority of the less abundant RNA fragments (> $40 \mathrm{nt}$ ) mapped onto ribosomal RNAs (Online Resource 20). However, the composition of the highly concentrated sRNA ( $<40 \mathrm{nt})$ was more complex. Size distribution analysis of this fraction showed abundant sequences of 21-22 nt length in CTL-sRNA-PT sample, corresponding to miRNAs. In contrast, species of 32-33 nt were the most abundant fragments in HD-sRNA-PT and mapped onto tRNAs (Fig. 6a). To characterize the composition of sRNA $<40 \mathrm{nt}$ we used the SeqCluster bioinformatic tool [53] that identifies clusters of coexpressed sRNA consistently and non-redundantly mapping onto the same precursor. Around 1000 clusters were identified in CTL- and HD-sRNA-PT samples (Online Resource 21), most of which mapped onto miRNAs and snoRNAs, followed by tRNA and gene fragments (Fig. 6b) (Online Resource 22). Regarding the relative abundance of annotated reads within each sample, miRNA-clusters were the most abundant type in CTL-sRNA-PT, whereas tRNA clusters were the most abundant representatives in HD-sRNA-PT (Fig. 6c). The analysis of the tRNA clusters showed abundant short sequences specifically mapping onto particular regions of different tRNAs, with this defining tRNA fragments (tRFs) derived from the $5^{\prime}$ - or $3^{\prime}$-end of the tRNAs (5'-tRFs and/or 3'-tRFs, respectively). Our analysis allowed us to identify specific tRFs overrepresented in HD-sRNA-PT compared to CTL-sRNA-PT with Ala-, Gly- and Val-5'tRFs showing the highest fold change (Fig. 6d, e). Increased expression of diverse tRFs was also detected in the HD-sRNA-CTX and HD-sRNA-CB, but the fold change was moderate compared with HD-sRNAPT (Fig. 6d). These data are in line with previous evidence showing activation of tRNA fragmentation under stress conditions linked to neurodegeneration [7, 22, 54].

Because diverse HD-sRNA-PT-overexpressed 5'tRFs halves have been shown to regulate the neural response to stress [7, 22, 31], we evaluated if specific tRFs overrepresented in HD-sRNA-PT were mediating neurotoxicity, employing the in vitro approach, as shown in Fig. $6 \mathrm{f}$. Primary striatal neurons at 7 DIV were exposed to sense (SS) and anti-sense (AS) versions of 5'tRF-Ala, 5'tRFVal, and 5'tRF-Gly, and cell viability was assessed using the MTS assay (Fig. 6e). $\mathrm{H}_{2} \mathrm{O}_{2}$ treatment was included as a positive control. Treatment with 5 'tRF-Ala SS resulted in decreased cell viability (Fig. 6f). In summary, our results show that particular tRFs are overexpressed in HD 
o Vehicle $\square$ HD-sRNA-PT+LNA-SCB $\square$ HD-sRNA-PT+LNA-CTG

a
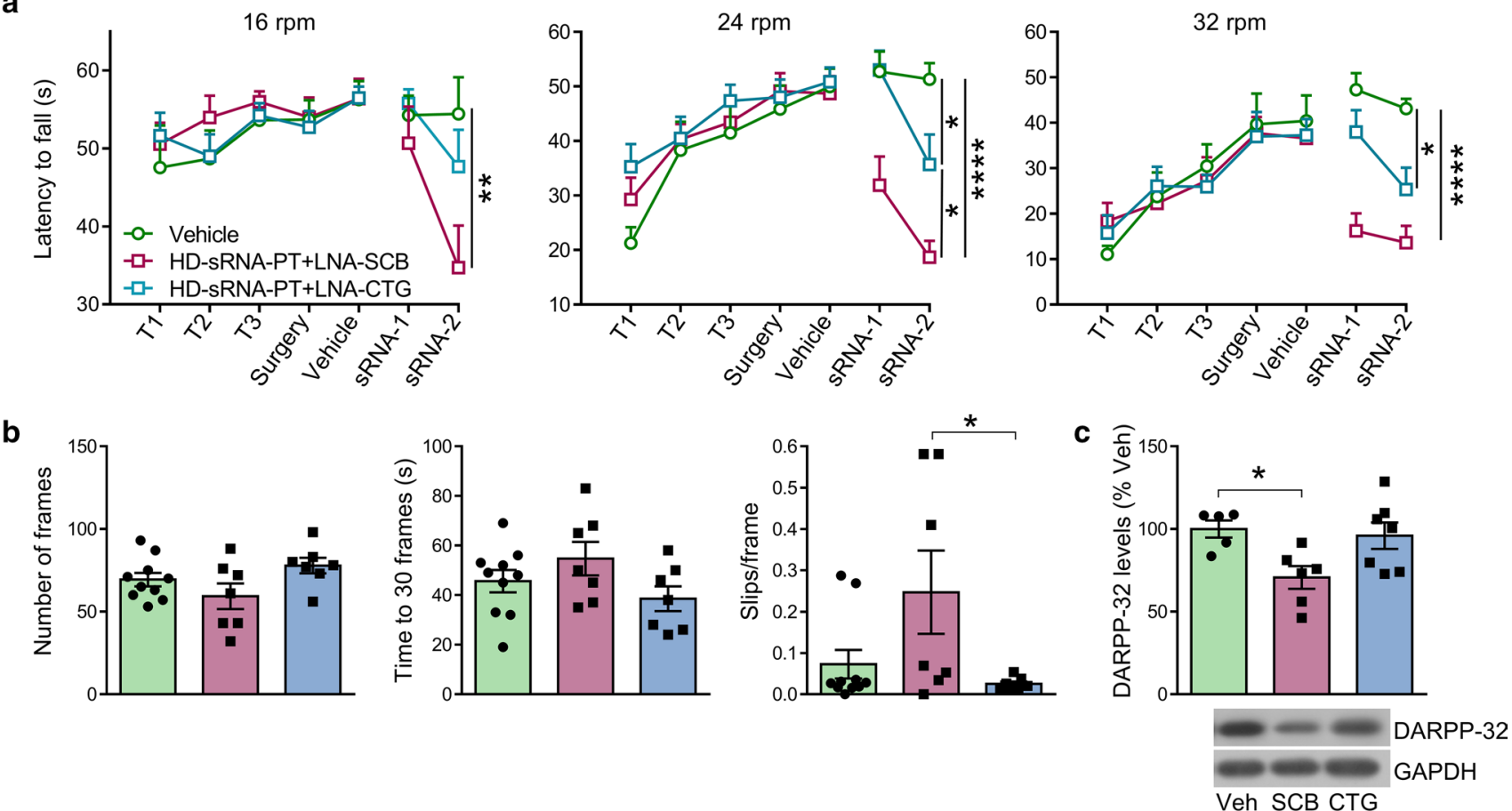

\section{d}
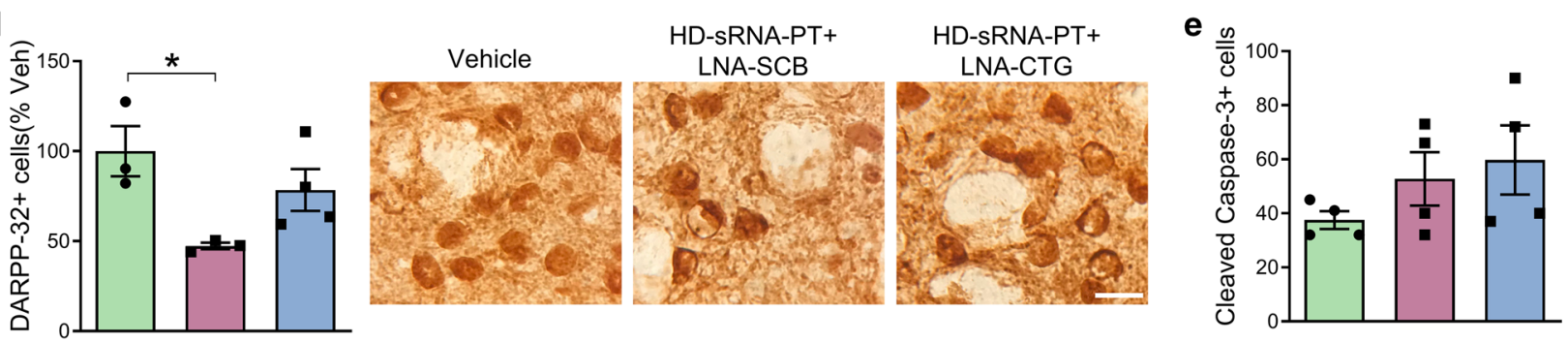

f
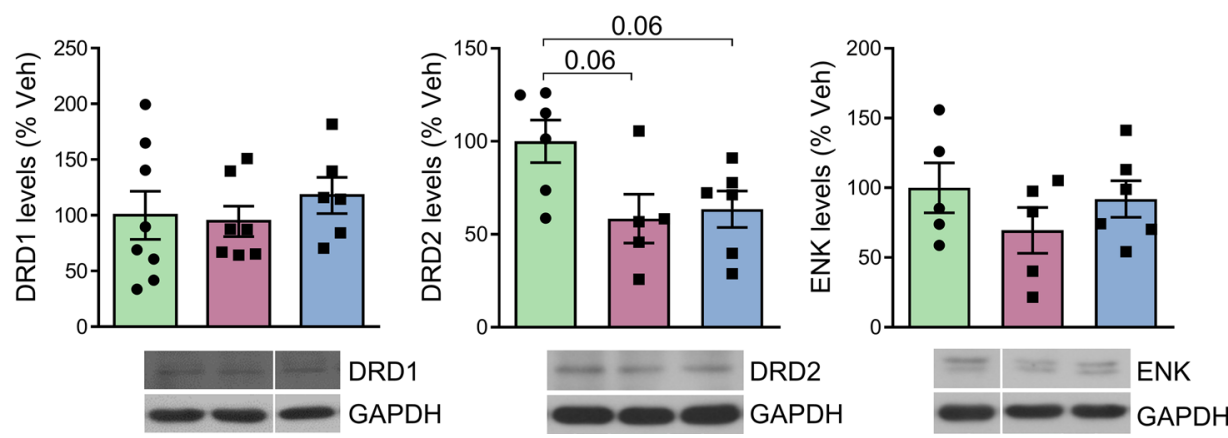

putamen and suggest that specific species are toxic for striatal neurons, suggesting that diverse classes of sRNA contribute to the overall toxicity induced by HD-sRNA.

\section{Discussion}

Important gaps remain in understanding the molecular basis underlying neurodegeneration in HD. In addition to the disruption of multiple cell processes induced by $\mathrm{mHTT}$ protein with expanded polyQ, others and we have provided evidence that transcribed CAG repeat RNAs directly participate in 
४Fig. 5 Blockage of sCAG from HD-sRNA-PT partially reduces motor alterations in WT mice. a Motor performance in the rotarod test. Vehicle $n=8$; HD-sRNA-PT+LNA-SCB $n=8$; HD-sRNAPT + LNA-CTG $n=8$. b Motor coordination in the balance beam test. Vehicle $n=10 ;$ HD-sRNA-PT + LNA-SCB $n=7$; HD-sRNA-PT+LNA-CTG $n=7$. c Protein levels of DARPP-32 were analyzed by Western Blot in striatal lysates. Vehicle $n=5$; HD-sRNA-PT + LNA-SCB $\quad n=6 ; \quad$ HD-sRNA-PT + LNA-CTG $n=7$. Representative immunoblot is shown. d Evaluation of striatal DARPP-32 positive cells. Vehicle $n=3$; HD-sRNA-PT + LNA-SCB $n=3$; HD-sRNA-PT+LNA-CTG $n=4$. Representative images are shown. Scale bar: $20 \mu \mathrm{m}$. e Graph showing the number of cleaved caspase-3-positive cells. Vehicle $n=4$; CTL-sRNA-PT + LNA-SCB $n=4$; HD-sRNA-PT + LNA-CTG $n=3$. f Protein levels of DRD1, DRD2 and enkephalin were analyzed by Western blot in striatal lysates. In the representative immunoblots, samples were run on the same gel, but lanes were noncontiguous (white line). Vehicle $n=5-6$; HD-sRNA-PT + LNA-SCB $n=5$; HD-sRNA-PT + LNA-CTG $n=6$. In $\mathbf{a}$, values represent the latency to fall as the mean \pm SEM. In $\mathbf{b}-\mathbf{f}$, each point represents data from an individual mouse. Data are shown as mean \pm SEM. $* * * * P<0.0001, * * P<0.01, * P<0.05$ versus all other groups; calculated by two-way ANOVA in a, and by one-way ANOVA in b-f. In all cases, Bonferroni was used as post hoc test

HD pathophysiology (for review, see [44]). Neurotoxicity produced by CAG repeat RNAs of different lengths may be complemented by detrimental effects of strongly perturbed sRNA and RAN translation products, thus adding complexity to the mechanisms causing neurodegeneration. Although conflicting observations have been reported regarding the neurotoxic activity of RAN translation in HD [3, 78], this discovery has hindered the differentiation between the toxicity produced by CAG repeats-containing RNA and RAN polypeptides.

Here, we designed a strategy to analyze the pathogenic potential of sRNA directly derived from the putamen of HD patients. Our data show that HD-sRNA-PT, when injected in the striatum, are sufficient to produce HD-like motor and molecular perturbations in naïve mice. Furthermore, our results suggest that sRNA from the most affected brain regions (putamen and cortex) are more toxic compared with sRNA from less-affected brain areas (cerebellum) [21]. HD-sRNA-PT induced motor alterations and gene expression changes that reflected neuronal dysfunction and immune activation. HD-sRNA-PT downregulated genes were enriched in neuronal pathways, including synaptic transmission, axon guidance, and nervous system development. Furthermore, the group of downregulated genes highlighted perturbations analogous to those found in HD mouse models and patients [1, 5, 8, 13, 20, 24, 25, 36, 38, 39, 51], strongly suggesting the participation of sRNA in the overall transcriptomic alterations associated with the disease. Loss of striatal neuronal markers was accompanied by a significant increase in cleaved caspase-3-positive cells, indicating activation of apoptotic cell death, which has been shown in the striatum of HD patients [56].
Importantly, downregulated transcripts induced by HD-sRNA-PT injection were specifically enriched in striatal MSNs of the indirect pathway with confirmed reduction of enkephalin and DRD2 protein levels. These results suggest a preferential detrimental activity of HD-sRNAPT on D2-MSNs, the striatal neuronal population to first degenerate in HD $[58,59]$. It has been speculated that cell-type-specific traits account for the differential modulation of toxicity in HD, including specific requirements for survival signaling, energy demand, neurochemical content, glutamate neurotransmission, and axonal transport [21]. Our data indicate that HD-sRNA-PT injection altered genes significantly related to glutamatergic synapse function and axon guidance, which could contribute to the impairment of pathways, especially relevant for striatal D2-MSNs normal function.

Transcripts upregulated by HD-sRNA-PT injection were enriched in immune response and inflammatory pathways. Although the pattern of cytokine expression favors a proinflammatory phenotype, no significant increases in Iba1 and GFAP levels were detected (data not shown). Glial activation might occur at later time-points, since recent studies on neuroinflammation show a sequential activation of microglial- and astrocyte-cytokine expression, preceding that of Iba- 1 and GFAP [50].

Our results point to a partial role of SCAG in the overall pathogenic alterations produced by HD-sRNA-PT injection into the mouse striatum. Motor perturbations were partially prevented by intrastriatal injection of LNA-CTG and the direct injection of pure sCAG RNAs in the striatum-induced moderate motor coordination deficits. sCAG derived from human HD brains may contribute to motor dysfunction by targeting DARPP-32 positive neurons, since LNA-CTG significantly prevented the loss of DARPP-32 levels induced by HD-sRNA-PT injection and pure sCAG infusion decreased striatal DARPP-32 protein levels. These results are in line with the detrimental effects of sCAG species in diverse cell models $[4,48]$ and reinforce the idea of sCAG RNAs as contributors to the disease. The neurotoxic activity of sCAG in cell models is dependent on the RISC gene-silencing machinery [4]. However, the present data suggest a post-transcriptional mechanism of action, since LNA-CTGs beneficial effects do not induce major changes in the transcriptome of HD-sRNA-injected striatum.

In addition, LNA-CTGs did not prevent HD-sRNAinduced decrease in the protein levels of diverse neuronal markers, nor in the glial reactive response, which agrees with the effect elicited by the injection of pure sCAG. Furthermore, the lack of effect of SCAG in the number of DARPP32 and cleaved caspase-3-positive cells suggests that sCAG do not contribute to the HD-sRNA-induced apoptotic cell death. In line with this, genetic mouse models expressing versions of the HTT exon-1 with expanded CAG repeats [75] 
a

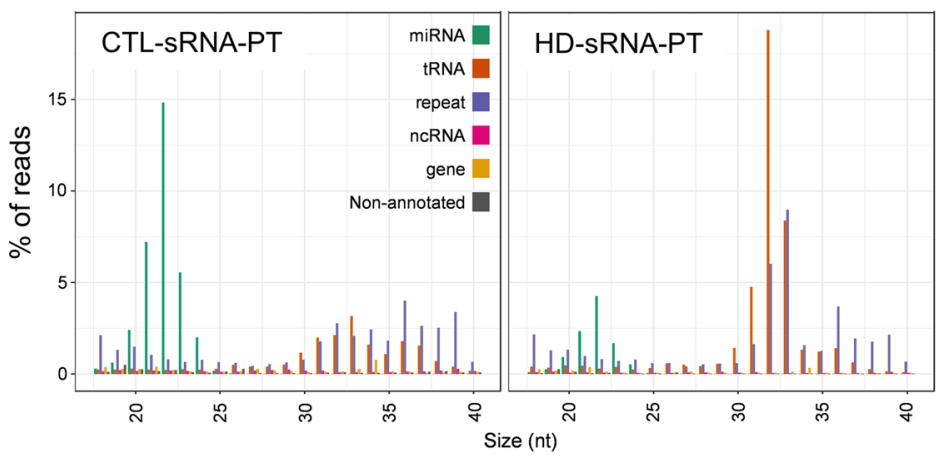

b

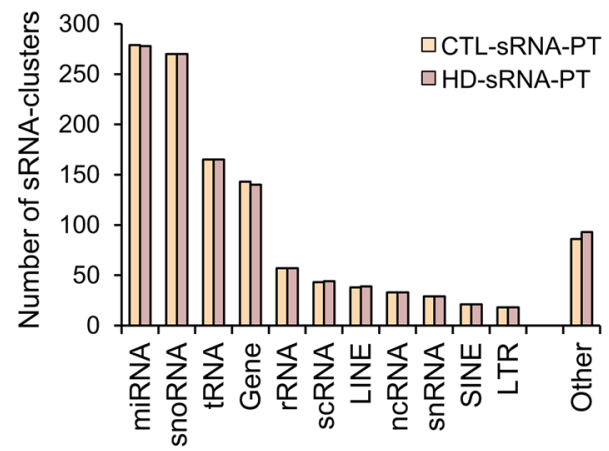

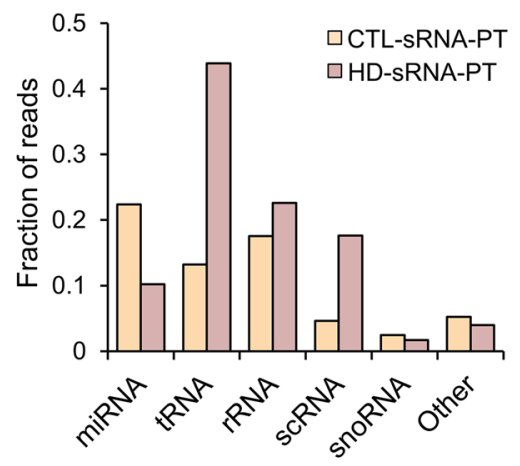

e

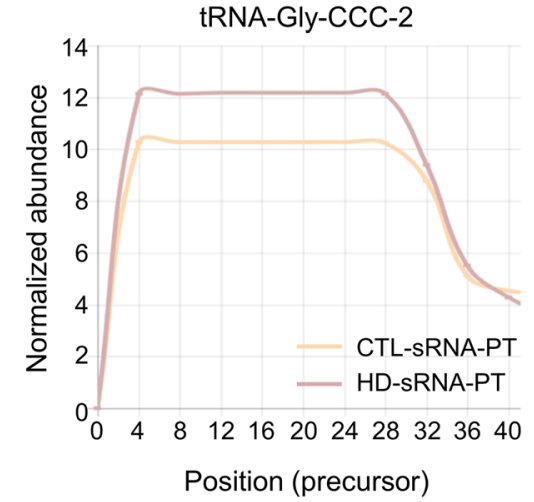

d
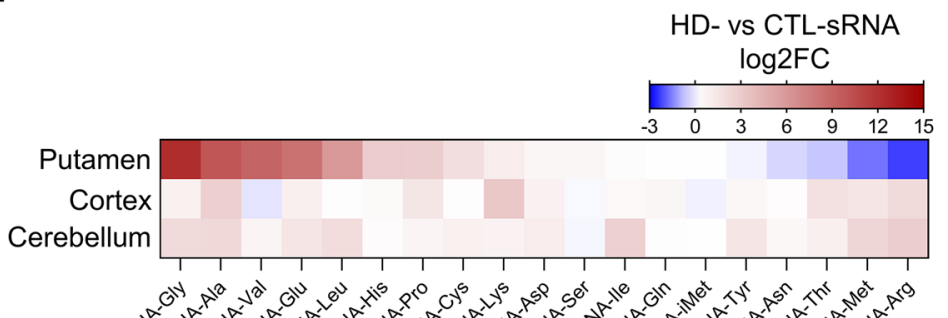

tRNA-Ala-CGC-3

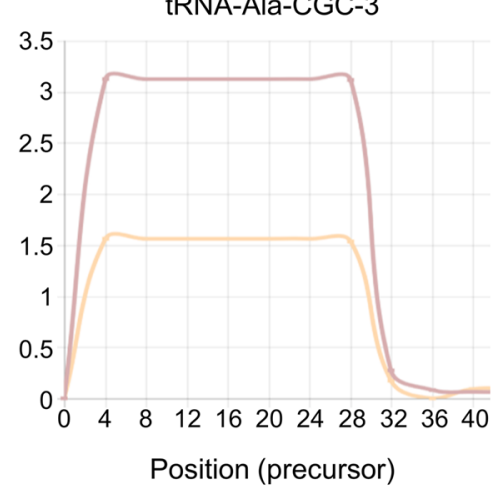

tRNA-Val-TAC-1

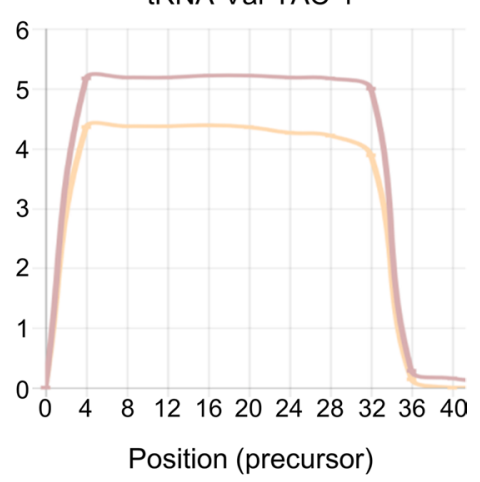

f

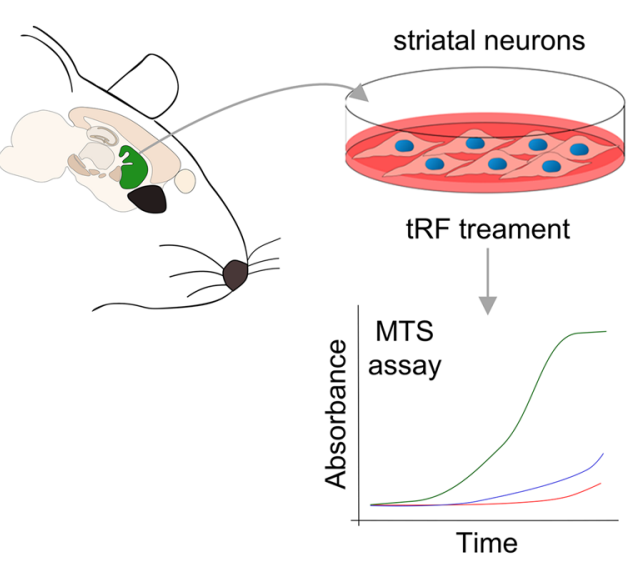

$\mathbf{f}$

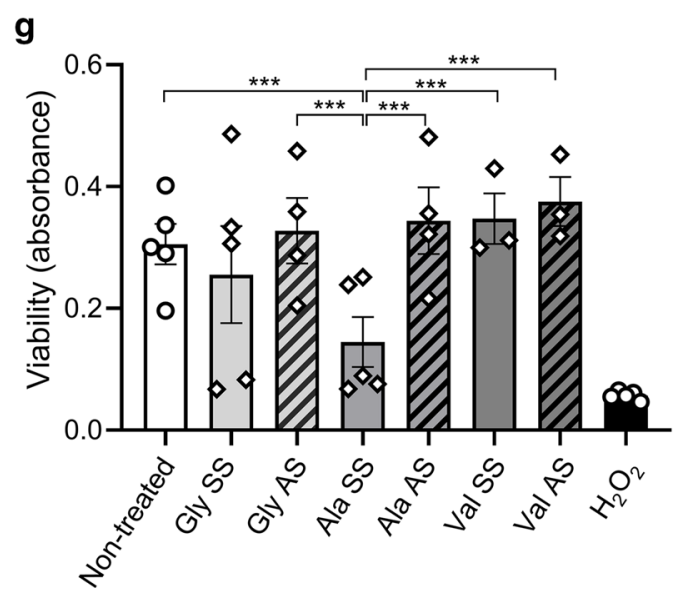


4Fig. 6 sRNA profiling reveals highly expressed tRFs in HD, with 5'tRF-Ala compromising neuronal viability. a CTL-sRNA-PT and HD-sRNA-PT ( $<40 \mathrm{nt}$ ) length distribution by class. Abundances (in $\%)$ are relative to all sequenced RNAs $(<40)$. b Absolute number of different classes of sRNA clusters. $\mathbf{c}$ Fraction of reads mapping onto a particular class of sRNA. d Heatmap of the log fold changes between HD- versus CTL-sRNA from putamen, cortex, and cerebellum. tRNA clusters mapping onto each tRNA isotype are shown. e Normalized abundance of sequences belonging to Gly-CCC-2-, Ala-CGC-3- and Val-TAC-1-tRNA clusters from CTL- and HD-sRNA-PT. f Schematic of experimental design to evaluate the effect of tRFs in neuronal viability: striatal primary neuronal cultures were exposed to sense (SS) and anti-sense (AS) versions of Gly-5'tRF, Ala-5'tRF, and Val-5'tRF at 7 days in vitro (DIV). Cell viability was measured using MTS assay $1 \mathrm{~h}$ after tRF treatment. $\mathrm{g}$ Graph showing neuronal viability determined by difference of absorbance $15 \mathrm{~min}$ and $3 \mathrm{~h}$ after MTS exposure in each well $\mathrm{H}_{2} \mathrm{O}_{2}$ was used as positive control. Non-treated $n=5$; Gly SS $n=5$; Gly AS $n=4$; Ala SS $n=6$; Ala AS $n=4$; Val SS $n=3$; Val AS $n=3 ; \mathrm{H}_{2} \mathrm{O}_{2} n=5$. Each point represents data from one independent culture. Data expressed as mean \pm SEM; $* * * P<0.001$ versus all other groups; calculated using a linear mixed-effects model with Bonferroni as post hoc test

or longer versions of the mutant $H T T$ gene $[26,57,79]$ differ in the severity and onset of the motor symptoms, but do not show prominent striatal cell death. We hypothesize that sRNA other than SCAG, produced in human HD putamen expressing full-length $\mathrm{mHTT}$, may participate in important HD pathological hallmarks, including striatal neuronal dysfunction and glial activation. This fits with the idea of a complex combination of pathogenic sRNA inducing toxicity.

The more likely detrimental species contributing to the HD-like neuropathology are those specifically expressed in the HD-sRNA-PT pool and virtually absent in CTL-sRNAPT pool. Sequencing analysis validated many of the previously reported HD overexpressed miRNAs, including miR196a-5p and miR-615-3p [27]. In addition, HD-sRNA-PT pool showed a strikingly over-representation of tRFs, which have been shown to accumulate by stress-induced cleavage of mature cytoplasmic tRNAs [65, 72] and can interfere with neuronal functions $[2,7,33,68]$. Our data show that a 5'-tRF derived from the tRNA-Ala-CGC-3 compromised cell viability in striatal primary neurons, suggesting that at least this sRNA contributes to HD-sRNA-PT pathogenicity. Nonetheless, additional studies are needed to determine whether tRFs are neurotoxic in vivo, in a model organism, and if their blockage could be further considered a therapeutic strategy for HD. Our data provide the basis for further investigations linking aberrant expression of diverse types of sRNA and HD neurodegeneration.

Along with the insights here provided, a limitation of the present study is the failure to consider a possible functional interaction of HD-sRNA with other detrimental players, including the expanded CAG repeats adopting a hairpin structure and the expanded polyQ. Furthermore, the real scenario likely involves temporal sRNA expression dynamics that our design, analyzing the effect of sRNA accumulated at advanced stages of the disease, does not contemplate. In line with this idea, expression profiling and functional screening of sRNA in disease evolution should help to elucidate if sRNA-mediated perturbations are an early phenomenon. Although we have observed a substantial and acute decline of striatal function in mice injected with HD-sRNA, additional studies to evaluate possible long-lasting detrimental effects of HD-sRNA-PT species should be performed.

In summary, the present study indicates that human brain HD-sRNA derived from the human putamen trigger motor coordination abnormalities in naïve mice and recapitulate major HD pathological hallmarks. The present results favor the idea that the orchestrated activity of sRNA, including sCAG, could be a causative factor in HD and further indicate that their blockage should be envisioned as a therapeutic strategy.

Supplementary Information The online version contains supplementary material available at https://doi.org/10.1007/s00401-021-02272-9.

Acknowledgements This work was supported by the Spanish government through the Spanish Ministry of Economy and Competitiveness (MINECO) and the Fondo Europeo de Desarrollo Regional (FEDER) (Project SAF2017-88452-R to EM and PID2019-106447RB-100 to EPN). We acknowledge support of the Spanish Ministry of Science Innovation and Universities through the "Maria Maeztu Unit of Excellence Program". We thank the staff of the Genomics Unit and the Bioinformatics unit at the CRG for RNA-seq performance and analysis, and A. López and M.T. Muñoz for their technical support. AGC is supported by a fellowship from the Fundación Tatiana Pérez de Guzmán el Bueno.

Author contributions JCM, AGC, VV, EPN, and EM designed the experimental approaches. JCM, AGC, VV, MGdH, MSB, AGV, CN, MM, FL, and DDL performed experiments. JCM, AGC, VV, LP, GE, $\mathrm{EPN}$, and EM analyzed and interpreted the data. All authors made intellectual contributions to the experimental design and discussion. EPN and EM coordinated the study, and JCM and EM wrote the manuscript.

\section{Compliance with ethical standards}

Conflict of interest The authors have declared that no conflict of interest exists.

\section{References}

1. Ament SA, Pearl JR, Cantle JP, Bragg RM, Skene PJ, Coffey SR et al (2018) Transcriptional regulatory networks underlying gene expression changes in Huntington's disease. Mol Syst Biol 14:e7435. https://doi.org/10.15252/msb.20167435

2. Anderson P, Ivanov $P$ (2014) tRNA fragments in human health and disease. FEBS Lett 588:4297-4304. https://doi.org/10.1016/j. febslet.2014.09.001

3. Bañez-Coronel M, Ayhan F, Tarabochia AD, Zu T, Perez BA, Tusi SK et al (2015) RAN translation in Huntington disease. Neuron 88:667-677. https://doi.org/10.1016/j.neuron.2015.10.038

4. Banez-Coronel M, Porta S, Kagerbauer B, Mateu-Huertas E, Pantano L et al (2012) A pathogenic mechanism in Huntington's 
disease involves small CAG-repeated RNAs with neurotoxic activity. PLoS Genet 8:e1002481. https://doi.org/10.1371/journ al.pgen.1002481

5. Becanovic K, Pouladi MA, Lim RS, Kuhn A, Pavlidis P, LuthiCarter R et al (2010) Transcriptional changes in Huntington disease identified using genome-wide expression profiling and cross-platform analysis. Hum Mol Genet 19:1438-1452. https:// doi.org/10.1093/hmg/ddq018

6. Bibb JA, Yan Z, Svenningsson P, Snyder GL, Pieribone VA, Horiuchi A et al (2000) Severe deficiencies in dopamine signaling in presymptomatic Huntington's disease mice. Proc Natl Acad Sci USA 97:6809-6814. https://doi.org/10.1073/pnas.120166397

7. Blanco S, Dietmann S, Flores JV, Hussain S, Kutter C, Humphreys $P$ et al (2014) Aberrant methylation of tRNAs links cellular stress to neuro-developmental disorders. EMBO J 33:2020-2039. https ://doi.org/10.15252/embj.201489282

8. Brochier C, Gaillard M-C, Diguet E, Caudy N, Dossat C, Segurens B et al (2008) Quantitative gene expression profiling of mouse brain regions reveals differential transcripts conserved in human and affected in disease models. Physiol Genom 33:170-179. https ://doi.org/10.1152/physiolgenomics.00125.2007

9. Creus-Muncunill J, Badillos-Rodríguez R, Garcia-Forn M, Masana M, Garcia-Díaz Barriga G, Guisado-Corcoll A et al (2019) Increased translation as a novel pathogenic mechanism in Huntington's disease. Brain 142:3158-3175. https://doi. org/10.1093/brain/awz230

10. van Dellen A, Welch J, Dixon RM, Cordery P, York D, Styles P et al (2000) $\mathrm{N}$-Acetylaspartate and DARPP-32 levels decrease in the corpus striatum of Huntington's disease mice. NeuroReport $11: 3751-3757$

11. Desplats PA, Kass KE, Gilmartin T, Stanwood GD, Woodward EL et al (2006) Selective deficits in the expression of striatal-enriched mRNAs in Huntington's disease. J Neurochem 96:743-757. https ://doi.org/10.1111/j.1471-4159.2005.03588.x

12. Dobin A, Davis CA, Schlesinger F, Drenkow J, Zaleski C et al (2013) STAR: ultrafast universal RNA-seq aligner. Bioinformatics 29:15-21. https://doi.org/10.1093/bioinformatics/bts635

13. Durrenberger PF, Fernando FS, Kashefi SN, Bonnert TP, Seilhean D, Nait-Oumesmar B et al (2015) Common mechanisms in neurodegeneration and neuroinflammation: a BrainNet Europe gene expression microarray study. J Neural Transm 122:1055-1068. https://doi.org/10.1007/s00702-014-1293-0

14. Ewels P, Magnusson M, Lundin S, Kaller M (2016) MultiQC: summarize analysis results for multiple tools and samples in a single report. Bioinformatics 32:3047-3048. https://doi.org/10.1093/ bioinformatics/btw354

15. Fourie C, Kim E, Waldvogel H, Wong JM, McGregor A, Faull RLM et al (2014) Differential changes in postsynaptic density proteins in postmortem Huntington's disease and Parkinson's disease human brains. J Neurodegener Dis 2014:938530. https://doi. org/10.1155/2014/938530

16. Francelle L, Galvan L, Brouillet E (2014) Possible involvement of self-defense mechanisms in the preferential vulnerability of the striatum in Huntington's disease. Front Cell Neurosci 8:1-13. https://doi.org/10.3389/fncel.2014.00295

17. Garcia-Alcalde F, Okonechnikov K, Carbonell J, Cruz LM, Gotz S, Tarazona S et al (2012) Qualimap: evaluating next-generation sequencing alignment data. Bioinformatics 28:2678-2679. https ://doi.org/10.1093/bioinformatics/bts503

18. García-Amado M, Prensa L (2012) Stereological analysis of neuron, glial and endothelial cell numbers in the human amygdaloid complex. PLoS ONE 7:e38692. https://doi.org/10.1371/journ al.pone. 0038692

19. García-Cabezas MÁ, John YJ, Barbas H, Zikopoulos B (2016) Distinction of neurons, glia and endothelial cells in the cerebral cortex: an algorithm based on cytological features. Front Neuroanat 10:107. https://doi.org/10.3389/fnana.2016.00107

20. Le Gras S, Keime C, Anthony A, Lotz C, De Longprez L, Brouillet E et al (2017) Altered enhancer transcription underlies Huntington's disease striatal transcriptional signature. Sci Rep 7:42875. https://doi.org/10.1038/srep42875

21. Han I, You Y, Kordower JH, Brady ST, Morfini GA (2010) Differential vulnerability of neurons in Huntington's disease: the role of cell type-specific features. J Neurochem 113:1073-1091. https ://doi.org/10.1111/j.1471-4159.2010.06672.x

22. Hanada T, Weitzer S, Mair B, Bernreuther C, Wainger BJ, Ichida J et al (2013) CLP1 links tRNA metabolism to progressive motorneuron loss. Nature 495:474-480. https://doi.org/10.1038/natur e11923

23. HDCRG (1993) A novel gene containing a trinucleotide repeat that is expanded and unstable on Huntington's disease chromosomes. The Huntington's Disease Collaborative Research Group. Cell 72:971-983

24. Hervas-Corpion I, Guiretti D, Alcaraz-Iborra M, Olivares R, Campos-Caro A, Barco A et al (2018) Early alteration of epigeneticrelated transcription in Huntington's disease mouse models. Sci Rep 8:9925. https://doi.org/10.1038/s41598-018-28185-4

25. Hodges A, Strand AD, Aragaki AK, Kuhn A, Sengstag T, Hughes $G$ et al (2006) Regional and cellular gene expression changes in human Huntington's disease brain. Hum Mol Genet 15:965-977. https://doi.org/10.1093/hmg/dd1013

26. Hodgson JG, Agopyan N, Gutekunst CA, Leavitt BR, LePiane F, Singaraja R et al (1999) A YAC mouse model for Huntington's disease with full-length mutant huntingtin, cytoplasmic toxicity, and selective striatal neurodegeneration. Neuron 23:181-192

27. Hoss AG, Kartha VK, Dong X, Latourelle JC, Dumitriu A, Hadzi TC et al (2014) MicroRNAs located in the Hox gene clusters are implicated in Huntington's disease pathogenesis. PLoS Genet 10:e1004188. https://doi.org/10.1371/journal.pgen.1004188

28. Huang DW, Sherman BT, Lempicki RA (2009) Systematic and integrative analysis of large gene lists using DAVID bioinformatics resources. Nat Protoc 4:44-57. https://doi.org/10.1038/nprot .2008 .211

29. Huang DW, Sherman BT, Lempicki RA (2009) Bioinformatics enrichment tools: paths toward the comprehensive functional analysis of large gene lists. Nucleic Acids Res 37:1-13. https:// doi.org/10.1093/nar/gkn923

30. Huber W, Carey VJ, Gentleman R, Anders S, Carlson M, Carvalho BS et al (2015) Orchestrating high-throughput genomic analysis with bioconductor. Nat Methods 12:115-121. https:// doi.org/10.1038/nmeth.3252

31. Ivanov P, O'Day E, Emara MM, Wagner G, Lieberman J, Anderson P (2014) G-quadruplex structures contribute to the neuroprotective effects of angiogenin-induced tRNA fragments. Proc Natl Acad Sci USA 111:18201-18206. https://doi.org/10.1073/ pnas. 1407361111

32. Jones TR, Kang IH, Wheeler DB, Lindquist RA, Papallo A, Sabatini DM et al (2008) Cell Profiler Analyst: data exploration and analysis software for complex image-based screens. BMC Bioinform 9:482. https://doi.org/10.1186/1471-2105-9-482

33. Karaca E, Weitzer S, Pehlivan D, Shiraishi H, Gogakos T, Hanada $\mathrm{T}$ et al (2014) Human CLP1 mutations alter tRNA biogenesis, affecting both peripheral and central nervous system function. Cell 157:636-650. https://doi.org/10.1016/j.cell.2014.02.058

34. Krol J, Fiszer A, Mykowska A, Sobczak K, de Mezer M, Krzyzosiak WJ (2007) Ribonuclease dicer cleaves triplet repeat hairpins into shorter repeats that silence specific targets. Mol Cell 25:575-586. https://doi.org/10.1016/j.molcel.2007.01.031

35. Krzyzosiak WJ, Sobczak K, Wojciechowska M, Fiszer A, Mykowska A et al (2012) Triplet repeat RNA structure and its 
role as pathogenic agent and therapeutic target. Nucleic Acids Res 40:11-26. https://doi.org/10.1093/nar/gkr729

36. Labadorf A, Hoss AG, Lagomarsino V, Latourelle JC, Hadzi TC, Bregu J et al (2015) RNA sequence analysis of human huntington disease brain reveals an extensive increase in inflammatory and developmental gene expression. PLoS ONE 10:e0143563. https ://doi.org/10.1371/journal.pone.0143563

37. Labbadia J, Morimoto RI (2013) Huntington's disease: underlying molecular mechanisms and emerging concepts. Trends Biochem Sci 38:378-385. https://doi.org/10.1016/j.tibs.2013.05.003

38. Langfelder P, Cantle JP, Chatzopoulou D, Wang N, Gao F, AlRamahi I et al (2016) Integrated genomics and proteomics define huntingtin CAG length-dependent networks in mice. Nat Neurosci 19:623-633. https://doi.org/10.1038/nn.4256

39. Langfelder P, Gao F, Wang N, Howland D, Kwak S, Vogt TF et al (2018) MicroRNA signatures of endogenous Huntington CAG repeat expansion in mice. PLoS ONE 13:1-20. https://doi. org/10.1371/journal.pone.0190550

40. Li L-B, Yu Z, Teng X, Bonini NM (2008) RNA toxicity is a component of ataxin-3 degeneration in Drosophila. Nature 453:1107-1111. https://doi.org/10.1038/nature06909

41. Livak KJ, Schmittgen TD (2001) Analysis of relative gene expression data using real-time quantitative PCR and the 2(Delta Delta C(T)) method. Methods 25:402-408. https://doi. org/10.1006/meth.2001.1262

42. Love MI, Huber W, Anders S (2014) Moderated estimation of fold change and dispersion for RNA-seq data with DESeq2. Genome Biol 15:550. https://doi.org/10.1186/s1305 9-014-0550-8

43. Luthi-Carter R, Strand A, Peters NL, Solano SM, Hollingsworth ZR, Menon AS et al (2000) Decreased expression of striatal signaling genes in a mouse model of Huntington's disease. Hum Mol Genet 9:1259-1271

44. Marti E (2016) RNA toxicity induced by expanded CAG repeats in Huntington's disease. Brain Pathol 26:779-786. https://doi. org/10.1111/bpa.12427

45. Martí E, Pantano L, Bañez-Coronel M, Llorens F, Miñones-Moyano E, Porta S et al (2010) A myriad of miRNA variants in control and Huntington's disease brain regions detected by massively parallel sequencing. Nucleic Acids Res 38:7219-7235. https://doi. org/10.1093/nar/gkq575

46. Martin M (2013) Cutadapt removes adapter sequences from highthroughput sequencing reads kenkyuhi hojokin gan rinsho kenkyu jigyo. EMBnet J 17:10-12. https://doi.org/10.14806/ej.17.1.200

47. Merienne N, Meunier C, Schneider A, Seguin J, Nair SS, Rocher $\mathrm{AB}$ et al (2019) Cell-type-specific gene expression profiling in adult mouse brain reveals normal and disease-state signatures. Cell Rep 26:2477-2493.e9. https://doi.org/10.1016/j.celre p.2019.02.003

48. Murmann AE, Gao QQ, Putzbach WE, Patel M, Bartom ET, Law CY et al (2018) Small interfering RNAs based on Huntingtin trinucleotide repeats are highly toxic to cancer cells. EMBO Rep. https://doi.org/10.15252/embr.201745336

49. Mykowska A, Sobczak K, Wojciechowska M, Kozlowski P, Krzyzosiak WJ (2011) CAG repeats mimic CUG repeats in the misregulation of alternative splicing. Nucleic Acids Res 39:8938-8951. https://doi.org/10.1093/nar/gkr608

50. Norden DM, Trojanowski PJ, Villanueva E, Navarro E, Godbout JP (2016) Sequential activation of microglia and astrocyte cytokine expression precedes increased Iba-1 or GFAP immunoreactivity following systemic immune challenge. Glia 64:300-316. https://doi.org/10.1002/glia.22930

51. Novati A, Hentrich T, Wassouf Z, Weber JJ, Yu-Taeger L, Deglon $\mathrm{N}$ et al (2018) Environment-dependent striatal gene expression in the BACHD rat model for Huntington disease. Sci Rep 8:5803. https://doi.org/10.1038/s41598-018-24243-z
52. Pantano L, Estivill X, Marti E (2010) SeqBuster, a bioinformatic tool for the processing and analysis of small RNAs datasets, reveals ubiquitous miRNA modifications in human embryonic cells. Nucleic Acids Res 38:e34. https://doi.org/10.1093/nar/ gkp1127

53. Pantano L, Estivill X, Marti E (2011) A non-biased framework for the annotation and classification of the non-miRNA small RNA transcriptome. Bioinformatics 27:3202-3203. https://doi. org/10.1093/bioinformatics/btr527

54. Pantano L, Friedlander MR, Escaramis G, Lizano E, PallaresAlbanell J, Ferrer I et al (2016) Specific small-RNA signatures in the amygdala at premotor and motor stages of Parkinson's disease revealed by deep sequencing analysis. Bioinformatics 32:673681. https://doi.org/10.1093/bioinformatics/btv632

55. Patro R, Mount SM, Kingsford C (2014) Sailfish enables alignment-free isoform quantification from RNA-seq reads using lightweight algorithms. Nat Biotechnol 32:462-464. https://doi. org/10.1038/nbt.2862

56. Pattison LR, Kotter MR, Fraga D, Bonelli RM (2006) Apoptotic cascades as possible targets for inhibiting cell death in Huntington's disease. J Neurol 253:1137-1142. https://doi.org/10.1007/ s00415-006-0198-8

57. Reddy PH, Williams M, Charles V, Garrett L, Pike-Buchanan L, Whetsell WOJ et al (1998) Behavioural abnormalities and selective neuronal loss in HD transgenic mice expressing mutated full-length HD cDNA. Nat Genet 20:198-202. https://doi. org/10.1038/2510

58. Richfield EK, Maguire-Zeiss KA, Vonkeman HE, Voorn P (1995) Preferential loss of preproenkephalin versus preprotachykinin neurons from the striatum of Huntington's disease patients. Ann Neurol 38:852-861. https://doi.org/10.1002/ana.410380605

59. Rikani AA, Choudhry Z, Choudhry AM, Rizvi N, Ikram H, Mobassarah NJ et al (2014) The mechanism of degeneration of striatal neuronal subtypes in Huntington disease. Ann Neurosci 21:112-114. https://doi.org/10.5214/ans.0972.7531.210308

60. Rosas HD, Koroshetz WJ, Chen YI, Skeuse C, Vangel M, Cudkowicz ME et al (2003) Evidence for more widespread cerebral pathology in early HD: an MRI-based morphometric analysis. Neurology 60:1615-1620

61. Rue L, Banez-Coronel M, Creus-Muncunill J, Giralt A, AlcalaVida R, Mentxaka G et al (2016) Targeting CAG repeat RNAs reduces Huntington's disease phenotype independently of huntingtin levels. J Clin Investig 126:4319-4330. https://doi.org/10.1172/ JCI83185

62. Rué L, López-Soop G, Gelpi E (2013) Brain region-and agedependent dysregulation of p62 and NBR1 in a mouse model of Huntington's disease. Neurobiol Dis 52:219-228

63. Saavedra A, García-Martínez JM, Xifró X, Giralt A, Torres-Peraza JF, Canals JM et al (2010) PH domain leucine-rich repeat protein phosphatase 1 contributes to maintain the activation of the PI3K/ Akt pro-survival pathway in Huntington's disease striatum. Cell Death Differ 17:324-335. https://doi.org/10.1038/cdd.2009.127

64. Saavedra A, Giralt A, Rué L, Xifró X, Xu J, Ortega Z et al (2011) Striatal-enriched protein tyrosine phosphatase expression and activity in Huntington's disease: a STEP in the resistance to excitotoxicity. J Neurosci 31:8150-8162

65. Saikia M, Krokowski D, Guan B-J, Ivanov P, Parisien M, Hu G et al (2012) Genome-wide identification and quantitative analysis of cleaved tRNA fragments induced by cellular stress. J Biol Chem 287:42708-42725. https://doi.org/10.1074/jbc.M112.37179

66. Sapp E, Kegel KB, Aronin N, Hashikawa T, Uchiyama Y, Tohyama K et al (2001) Early and progressive accumulation of reactive microglia in the Huntington disease brain. J Neuropathol Exp Neurol 60:161-172 
67. Sathasivam K, Neueder A, Gipson TA, Landles C, Benjamin AC, Bondulich MK et al (2013) Aberrant splicing of HTT generates the pathogenic exon 1 protein in Huntington disease. Proc Natl Acad Sci USA 110:2366-2370. https://doi.org/10.1073/ pnas. 1221891110

68. Schaffer AE, Eggens VRC, Caglayan AO, Reuter MS, Scott E, Coufal NG et al (2014) CLP1 founder mutation links tRNA splicing and maturation to cerebellar development and neurodegeneration. Cell 157:651-663. https://doi.org/10.1016/j.cell.2014.03.049

69. Schmieder R, Lim YW, Edwards R (2012) Identification and removal of ribosomal RNA sequences from metatranscriptomes. Bioinformatics 28:433-435. https://doi.org/10.1093/bioinforma tics/btr669

70. Steibel JP, Poletto R, Coussens PM, Rosa GJM (2009) A powerful and flexible linear mixed model framework for the analysis of relative quantification RT-PCR data. Genomics 94:146-152. https ://doi.org/10.1016/j.ygeno.2009.04.008

71. Swinnen B, Robberecht W, Van Den Bosch L (2020) RNA toxicity in non-coding repeat expansion disorders. EMBO J 39:e101112. https://doi.org/10.15252/embj.2018101112

72. Thompson DM, Lu C, Green PJ, Parker R (2008) tRNA cleavage is a conserved response to oxidative stress in eukaryotes. RNA 14:2095-2103. https://doi.org/10.1261/rna.1232808

73. Torres-Peraza JF, Giralt A, Garcia-Martinez JM, Pedrosa E, Canals JM, Alberch J (2008) Disruption of striatal glutamatergic transmission induced by mutant huntingtin involves remodeling of both postsynaptic density and NMDA receptor signaling. Neurobiol Dis 29:409-421. https://doi.org/10.1016/j.nbd.2007.10.003
74. Tsoi H, Lau TC-K, Tsang S-Y, Lau K-F, Chan HYE (2012) CAG expansion induces nucleolar stress in polyglutamine diseases. Proc Natl Acad Sci USA 109:13428-13433. https://doi.org/10.1073/ pnas. 1204089109

75. Turmaine M, Raza A, Mahal A, Mangiarini L, Bates GP, Davies SW (2000) Nonapoptotic neurodegeneration in a transgenic mouse model of Huntington's disease. Proc Natl Acad Sci USA 97:80938097. https://doi.org/10.1073/pnas.110078997

76. Vonsattel JP, DiFiglia M (1998) Huntington disease. J Neuropathol Exp Neurol 57:369-384

77. Wang L-C, Chen K-Y, Pan H, Wu C-C, Chen P-H, Liao Y-T et al (2011) Muscleblind participates in RNA toxicity of expanded CAG and CUG repeats in Caenorhabditis elegans. Cell Mol Life Sci 68:1255-1267. https://doi.org/10.1007/s00018-010-0522-4

78. Yang S, Yang H, Huang L, Chen L, Qin Z, Li S et al (2020) Lack of RAN-mediated toxicity in Huntington's disease knock-in mice. Proc Natl Acad Sci USA 117:4411-4417. https://doi.org/10.1073/ pnas.1919197117

79. Yu Z-X, Li S-H, Evans J, Pillarisetti A, Li H, Li X-J (2003) Mutant huntingtin causes context-dependent neurodegeneration in mice with Huntington's disease. J Neurosci 23:2193-2202

Publisher's Note Springer Nature remains neutral with regard to jurisdictional claims in published maps and institutional affiliations. 